\title{
Central limit theorem for Bose gases interacting through singular potentials
}

\author{
Simone Rademacher ${ }^{1,2}$
}

Received: 18 September 2019 / Revised: 27 February 2020 / Accepted: 3 March 2020 /

Published online: 12 March 2020

(c) The Author(s) 2020

\begin{abstract}
We consider a system of $N$ bosons in the limit $N \rightarrow \infty$, interacting through singular potentials. For initial data exhibiting Bose-Einstein condensation, the many-body time evolution is well approximated through a quadratic fluctuation dynamics around a cubic nonlinear Schrödinger equation of the condensate wave function. We show that these fluctuations satisfy a (multi-variate) central limit theorem.
\end{abstract}

Keywords Many-body quantum mechanics · Central limit theorem · Bogoliubov transformation $\cdot$ Bose-Einstein condensate

Mathematics Subject Classification 35Q40 $\cdot 35 \mathrm{Q} 55 \cdot 81 \mathrm{Q} 05 \cdot 82 \mathrm{C} 10$

\section{Introduction}

We consider a system of $N$ bosons with Hamilton operator

$$
H_{N}=\sum_{j=1}^{N}-\Delta_{x_{j}}+\frac{1}{N} \sum_{1 \leq j<k \leq N} V_{N}\left(x_{j}-x_{k}\right)
$$

acting on $L_{s}^{2}\left(\mathbb{R}^{3 N}\right)$, the subspace of $L^{2}\left(\mathbb{R}^{3 N}\right)$ consisting of functions which are symmetric with respect to permutations. The $N$-dependent two-body interaction potential is given through

Simone Rademacher acknowledges partial support from the NCCR SwissMAP. This project has received funding from the European Union's Horizon 2020 research and innovation program under the Marie Skłodowska-Curie Grant Agreement No. 754411.

$凶 \quad$ Simone Rademacher

simone.rademacher@ist.ac.at

1 Institute of Mathematics, University of Zurich, Winterthurerstrasse 190, 8057 Zurich, Switzerland

2 Present Address: IST Austria, Am Campus 1, 3400 Klosterneuburg, Austria 


$$
V_{N}(x)=N^{3 \beta} V\left(N^{\beta} x\right)
$$

In the following, we assume $V \geq 0$ to be smooth, spherically symmetric, and compactly supported. For $\beta=0$, the Hamiltonian (1.1) describes the mean-field regime characterized by a large number of weak collisions, whereas for $\beta>1 / 3$ the collisions of the particles are rare but strong. In the Gross-Pitaevskii regime $(\beta=1)$, pair correlations play a crucial role. Here, we study intermediate regimes $\beta \in(0,1)$ in the limit $N \rightarrow \infty$ where the particles interact through singular potentials.

The time evolution is governed by the Schrödinger equation

$$
i \partial_{t} \psi_{N, t}=H_{N} \psi_{N, t} .
$$

For $\beta=0$ (mean-field regime), the solution of (1.2) can be approximated by products of solutions of the Hartree equation

$$
i \partial_{t} \varphi_{t}=-\Delta \varphi_{t}+\left(V *\left|\varphi_{t}\right|^{2}\right) \varphi_{t}
$$

with initial data $\varphi_{0} \in L^{2}\left(\mathbb{R}^{3}\right)$. See, for example, [1-5,10,16,20-22,25,26,34]. For $0<\beta \leq 1$, on the other hand, the solution $\psi_{N, t}$ of (1.2) can be approximated by the nonlinear Schrödinger equation

$$
i \partial_{t} \varphi_{t}=-\Delta \varphi_{t}+\sigma \varphi_{t}
$$

with $\sigma=\widehat{V}(0)$ if $\beta<1$ and $\sigma=8 \pi \mathfrak{a}_{0}$ if $\beta=1$ (Gross-Pitaevskii regime). Hereafter, $\mathfrak{a}_{0}$ denotes the scattering length associated with the potential $V$ defined through the solution of the zero-energy scattering equation

$$
\left[-\Delta+\frac{1}{2} V\right] f=0
$$

with boundary condition $f(x) \rightarrow 1$ as $|x| \rightarrow \infty$. Then, outside the support of $V$, the solution $f$ is given through

$$
f(x)=1-\mathfrak{a}_{0} /|x|,
$$

where $\mathfrak{a}_{0}$ is defined as the scattering length of the potential $V$. In [17-19], it is shown that if the one-particle reduced density $\gamma_{N}$ associated with $\psi_{N}$ satisfies

$$
\gamma_{N} \rightarrow\left|\varphi_{0}\right\rangle\left\langle\varphi_{0}\right|
$$

in the trace norm topology and

$$
\left\langle\psi_{N}, H_{N} \psi_{N}\right\rangle \leq C N
$$


then the one-particle reduced density $\gamma_{N, t}$ associated with the solution $\psi_{N, t}$ of (1.2) obeys

$$
\gamma_{N, t} \rightarrow\left|\varphi_{t}\right\rangle\left\langle\varphi_{t}\right|
$$

where $\varphi_{t}$ denotes the solution of (1.3). In fact, in [17] considering the case $\beta<1$, the energy condition (1.6) on the initial data is not needed. For more results in the GrossPitaevskii regime, see [7,12,15,30,31]. An overview on the derivation of the nonlinear Schrödinger equation from many-body quantum dynamics is given in $[8,23,33]$.

\subsection{Norm approximation}

Besides the convergence of the one-particle reduced density $\gamma_{N, t}$ associated with $\psi_{N, t}$, the norm approximation of $\psi_{N, t}$ has been studied for different settings of $\beta \in(0,1)$ in $[11,24,28,29]$. Our result is based on the norm approximation obtained in [11] covering $\beta<1$ whose ideas we explain in the following.

Truncated Fock space. As first step toward the norm approximation in [11], the contribution of the Bose-Einstein condensate is factored out. This is realized through the unitary $\mathscr{U}_{\varphi_{N, t}}: L_{S}\left(\mathbb{R}^{3 N}\right) \rightarrow \mathscr{F}_{\perp \varphi_{N, t}}$. It maps the $N$-particle sector of the bosonic Fock space

$$
\mathscr{F}=\bigoplus_{n \geq 0} L_{s}\left(\mathbb{R}^{3 n}\right)
$$

into the truncated Fock space

$$
\underset{F^{\prime} \varphi_{N, t}}{\leq N}=\bigoplus_{n=0}^{N} L_{\perp \varphi_{N, t}}^{2}\left(\mathbb{R}^{3}\right)^{\otimes_{s} n}
$$

defined over the orthogonal complement $L_{\perp \varphi_{N, t}}^{2}\left(\mathbb{R}^{3}\right)$ of the subspace of $L^{2}\left(\mathbb{R}^{3}\right)$ spanned by the condensate wave function $\varphi_{N, t}$. This unitary has first been used in [27] in the mean-field regime. Its definition is based on the observation that every $\psi_{N} \in L_{s}^{2}\left(\mathbb{R}^{3 N}\right)$ has a unique decomposition

$$
\psi_{N}=\sum_{n=0}^{N} \alpha^{(n)} \otimes_{S} \varphi_{N, t}^{N-n},
$$

where $\alpha^{(n)} \in L_{\perp \varphi_{N, t}}^{2}\left(\mathbb{R}^{3}\right)^{\otimes_{s} n}$ for all $n=1, \ldots, N$. Then,

$$
\mathscr{U}_{\varphi_{N, t}} \psi_{N}=\left\{\alpha^{(0)}, \alpha^{(1)}, \ldots, \alpha^{(n)}\right\}
$$

This unitary satisfies the following properties proven in [27] 


$$
\begin{aligned}
\mathscr{U}_{\varphi_{N, t}} a^{*}\left(\varphi_{N, t}\right) a\left(\varphi_{N, t}\right) \mathscr{U}_{\varphi_{N, t}}^{*} & =N-\mathscr{N}_{+}(t) \\
\mathscr{U}_{\varphi_{N, t}} a^{*}\left(\varphi_{N, t}\right) a(f) \mathscr{U}_{\varphi_{N, t}}^{*} & =\sqrt{N-\mathscr{N}_{+}(t) a(f)} \\
\mathscr{U}_{\varphi_{N, t}} a^{*}(f) a\left(\varphi_{N, t}\right) \mathscr{U}_{\varphi_{N, t}}^{*} & =a^{*}(f) \sqrt{N-\mathscr{N}_{+}(t)} \\
\mathscr{U}_{\varphi_{N, t}} a^{*}(f) a(g) \mathscr{U}_{\varphi_{N, t}}^{*} & =a^{*}(f) a(g)
\end{aligned}
$$

for all $f, g \in L_{\perp \varphi_{N, t}}^{2}\left(\mathbb{R}^{3}\right)$. Here $a^{*}(f), a(f)$ denote the standard creation and annihilation operators on the bosonic Fock space $\mathscr{F}$. On the truncated Fock space, we define modified creation and annihilation operators

$$
b^{*}(f)=a^{*}(f) \sqrt{\frac{N-\mathscr{N}_{+}(t)}{N}}, \quad b(f)=\sqrt{\frac{N-\mathscr{N}_{+}(t)}{N}} a(f) .
$$

The modified creation operator $b^{*}(f)$ excites one particle from the condensate into its complement, while $b(f)$ annihilates an excitation into the condensate. We define the vector $\xi_{N, t}:=\mathscr{U}_{\varphi_{N, t}} \psi_{N, t}$ representing the fluctuation outside the condensate and observe

$$
i \partial_{t} \xi_{N, t}=\mathscr{L}_{N, t} \xi_{N, t}, \quad \text { with } \quad \mathscr{L}_{N, t}=\mathscr{U}_{\varphi_{N, t}} H_{N} \mathscr{U}_{\varphi_{N, t}}^{*}+\left(i \partial_{t} \mathscr{U}_{\varphi_{N, t}}\right) \mathscr{U}_{\varphi_{N, t}}^{*}
$$

with initial data $\xi_{N, 0}=\mathscr{U}_{\varphi_{N, 0}} \psi_{N, 0}$.

From the truncated Fock space to the bosonic Fock space. We approximate the generator $\mathscr{L}_{N, t}$ acting on the truncated Fock space only with a modified generator $\widetilde{\mathscr{L}}_{N, t}$ defined on the whole bosonic Fock space. We consider regimes with a small number of excitations $\mathscr{N}_{+}(t)$. For this reason, we realize the approximation of $\mathscr{L}_{N, t}$ through $\widetilde{\mathscr{L}}_{N, t}$ by replacing $\sqrt{N-\mathscr{N}_{+}(t)}$ with $\sqrt{N} G_{M}\left(\mathscr{N}_{+}(t) / N\right)$, where

$$
G_{M}(\tau):=\sum_{n=0}^{M} \frac{(2 n) !}{(n !)^{2} 4^{n}(1-2 n)} t^{n}
$$

is the $M$ th Taylor polynom of $\sqrt{1-\tau}$ expanded at the point $\tau_{0}=0$. For a precise definition, see [11, eq. (54)].

Correlation structure through Bogoliubov transformation. In the intermediate regime, correlations are important (at least if $\beta>1 / 2$ ). For their implementation, we consider for fixed $\ell>0$ the ground state of the scattering equation

$$
\left[-\Delta+\frac{1}{2 N} V_{N}\right] f_{N}=\lambda_{N} f_{N}
$$

with Neumann boundary conditions on the ball $B_{\ell}(0)$. We fix $f_{N}(x)=1$ for all $|x|=\ell$ and extend $f_{N}$ to $\mathbb{R}^{3}$ by setting $f_{N}(x)=1$ for all $|x| \geq \ell$. 
In [10], the nonlinear Schrödinger equation (1.3) is replaced by the $N$-dependent Hartree equation

$$
i \partial_{t} \varphi_{N, t}=-\Delta \varphi_{N, t}+\left(V_{N} f_{N} *\left|\varphi_{N, t}\right|^{2}\right) \varphi_{N, t}
$$

with initial data $\varphi_{N, 0}=\varphi_{0}$ (the condensate wave function at time $t=0$ ) to approximate the time evolved condensate wave function. The well-posedness of (1.12) is shown in [10, Appendix B].

The correlation structure is implemented through the Bogoliubov transformation

$$
T_{N, t}=\exp \left(\frac{1}{2} \int \mathrm{d} x \mathrm{~d} y\left[\eta_{N, t}(x, y) a_{x} a_{y}-h . c .\right]\right),
$$

where $\eta_{N, t}$ denotes the Hilbert-Schmidt operator with integral kernel

$$
\eta_{N, t}(x ; y)=-\left(q_{N, t} \otimes q_{N, t}\right) N \omega_{N}(x-y) \varphi_{N, t}^{2}((x+y) / 2) .
$$

Here, $\omega_{N}=1-f_{N}$ and $\varphi_{N, t}$ are as defined in (1.11) resp. (1.12) and $q_{N, t}=1-$ $\left|\varphi_{N, t}\right\rangle\left\langle\varphi_{N, t}\right|$. The Bogoliubov transformation acts on the creation and annihilation operators as

$$
\begin{aligned}
T_{N, t} a(f) T_{N, t}^{*} & \left.=a\left(\cosh _{\eta_{N, t}}(f)\right)+a^{*}\left(\sinh _{\eta_{N, t}} \bar{f}\right)\right) \\
T_{N, t} a^{*}(f) T_{N, t}^{*} & =a^{*}\left(\cosh _{\eta_{N, t}}(f)\right)+a\left(\sinh _{\eta_{N, t}}(\bar{f})\right)
\end{aligned}
$$

for all $f \in L^{2}\left(\mathbb{R}^{3}\right)$. The operators $\sinh _{\eta_{N, t}}$ and $\cosh _{\eta_{N, t}}$ are defined through the absolutely convergent series of products of the operator $\eta_{N, t}$

$$
\cosh _{\eta_{N, t}}=\sum_{n \geq 0} \frac{1}{(2 n) !}\left(\eta_{N, t} \bar{\eta}_{N, t}\right)^{n}, \quad \sinh _{\eta_{N, t}}=\sum_{n \geq 0} \frac{1}{(2 n+1) !}\left(\eta_{N, t} \bar{\eta}_{N, t}\right)^{n} \eta_{N, t} .
$$

Let $\mathscr{G}_{N, t}$ be the generator given through

$$
\mathscr{G}_{N, t}=\left(i \partial_{t} T_{N, t}\right) T_{N, t}^{*}+T_{N, t} \tilde{\mathscr{L}}_{N, t} T_{N, t}^{*}
$$

In fact, the special choice of (1.11) and (1.12) allows crucial cancellations in the generator $\mathscr{G}_{N, t}$. Note that $\mathscr{G}_{N, t}$ consists of terms which are quadratic in creation and annihilation operators and of terms of higher order. Nevertheless, in [11, Lemma 5], it is shown that $\mathscr{G}_{N, t}$ can be approximated through the generator $\mathscr{G}_{2, N, t}$ containing quadratic terms only.

Limiting quadratic dynamics. We are interested in the limit $N \rightarrow \infty$ of $\mathscr{G}_{2, N, t}$ defined in (1.16). In order to replace the Bogoliubov transformation $T_{N, t}$ defined in (1.13) with a limiting one, we define the limiting kernel $\omega_{\infty}$ of $\omega_{N}$ through 


$$
\omega_{\infty}(x)=\frac{\mathfrak{b}_{0}}{8 \pi}\left[\frac{1}{|x|}-\frac{3}{2 \ell}+\frac{x^{2}}{3 \ell^{3}}\right]
$$

for $|x| \leq \ell$ and $\omega_{\infty}(x)=0$ otherwise. Here, we used the notation $\mathfrak{b}_{0}=\int \mathrm{d} x V(x)$.

Furthermore, the solution $\varphi_{N, t}$ of the modified Hartree equation (1.12) with initial data $\varphi_{0} \in H^{4}\left(\mathbb{R}^{3}\right)$ can be approximated with the solution $\varphi_{t}$ of (1.3) with $\sigma=\widehat{V}(0)$ and with initial data $\varphi_{0}$. To be more precise, [10, Proposition B.1] shows that there exists a constant $C>0$ (depending on $\left\|\varphi_{0}\right\|_{H^{4}}$ ) such that

$$
\left\|\varphi_{t}-\varphi_{N, t}\right\|_{2} \leq C N^{-\gamma} \exp (C \exp (C|t|))
$$

with $\gamma=\min \{\beta, 1-\beta\}$. Standard arguments (see, for example, [10, Proposition B.1]) imply that there exists a constant $C>0$ such that

$$
\left.\left.\left\|\varphi_{t}\right\|_{2} \leq C, \quad\left\|\varphi_{t}\right\|_{\infty} \leq C \exp (C|t|)\right), \quad\left\|\varphi_{t}\right\|_{H^{n}} \leq C \exp (C|t|)\right)
$$

for all $n \in \mathbb{N}$. The approximations (1.17) and (1.18) lead to a limiting kernel

$$
\eta_{t}(x ; y)=-\left(q_{t} \otimes q_{t}\right) \omega_{\infty}(x-y) \varphi_{t}^{2}((x+y) / 2) .
$$

We define the limiting Bogoliubov transformation

$$
T_{t}=\exp \left(\frac{1}{2} \int \mathrm{d} x \mathrm{~d} y\left[\eta_{t}(x, y) a_{x} a_{y}-h . c .\right]\right) .
$$

In fact, (1.17) and (1.18) yield that there exists a constant $C>0$ such that

$$
\left\|\eta_{N, t}-\eta_{t}\right\|_{2} \leq C N^{-\gamma} \exp (C \exp (C|t|))
$$

where $\gamma=\min \{\beta, 1-\beta\}$.

In order to define the limiting dynamics, we introduce some more notation. We use the shorthand notation $j_{x}(\cdot)=j(\cdot, x)$ for any $j \in L^{2}\left(\mathbb{R}^{3} \times \mathbb{R}^{3}\right)$. Furthermore, we decompose $\operatorname{sh}_{\eta_{t}}=\eta_{t}+\mathrm{r}_{t}, \mathrm{ch}_{\eta_{t}}=\mathbb{1}+\mathrm{p}_{t}$ and

$$
\eta_{t}(x ; y)=-\omega_{\infty}(x-y) \varphi_{t}^{2}((x+y) / 2)+\mu_{t}(x ; y)=k_{t}(x ; y)+\mu_{t}(x ; y)
$$

for all $x, y \in \mathbb{R}^{3}$.

A slight modification of the arguments in [10, Appendix C] shows some properties of the kernels. For these, we consider initial data $\varphi_{0} \in H^{4}\left(\mathbb{R}^{3}\right)$ of (1.3). There exist a constant $C>0$ (depending only on $\left\|\varphi_{0}\right\|_{H^{4}\left(\mathbb{R}^{3}\right)}$ and on $V$ ) such that on the one hand

$$
\left\|\mathrm{ch}_{\eta_{t}}\right\| \leq C, \quad \text { and } \quad\left\|k_{t}\right\|_{2},\left\|\eta_{t}\right\|_{2},\left\|\operatorname{sh}_{\eta_{t}}\right\|_{2},\left\|\mathrm{p}_{t}\right\|_{2},\left\|\mathrm{r}_{t}\right\|_{2},\left\|\mu_{t}\right\|_{2} \leq C
$$


where $\|\cdot\|$ denotes the operator norm. On the other hand, denoting with $\nabla_{1} k_{t}$ and $\nabla_{2} k_{t}$ the operator with the kernel $\nabla_{x} k_{t}(x ; y)$

$$
\left\|\partial_{t} \eta_{t}\right\|_{2} \leq C e^{C|t|}, \quad \max \left\{\sup _{x} \int \mathrm{d} z\left|\nabla_{1} k_{t}(x ; z)\right|, \sup _{y} \int \mathrm{d} z\left|\nabla_{1} k_{t}(z ; y)\right|\right\} \leq C .
$$

Furthermore, let $\Delta_{1} \mathrm{r}_{t}$ resp. $\Delta_{2} \mathrm{r}_{t}$ be the operator having the kernel $\Delta_{x} \mathrm{r}_{t}(x ; y)$ resp. $\Delta_{y} \mathrm{r}_{t}(x ; y)$, then for all $i=1,2$

$$
\left\|\Delta_{i} \mathrm{r}_{t}\right\|_{2},\left\|\Delta_{i} \mathrm{p}_{t}\right\|_{2},\left\|\Delta_{i} \mu_{t}\right\|_{2} \leq C e^{C|t|}
$$

In order to simplify notation, we write in the following $\mathrm{sh}_{\eta_{t}}=\mathrm{sh}, \mathrm{ch}_{\eta_{t}}=\mathrm{ch}$ resp. $\mathrm{r}_{t}=\mathrm{r}, \mathrm{k}_{t}=\mathrm{k}, \mathrm{p}_{t}=\mathrm{p}$.

Definition 1 We define the limiting dynamics $\mathscr{U}_{2}(t ; s)$ satisfying

$$
i \partial_{t} \mathscr{U}_{2}(t ; s)=\mathscr{G}_{2}(t) \mathscr{U}_{2}(t ; s) \text { and } \mathscr{U}_{2}(s ; s)=\mathbb{1}
$$

where $\mathscr{G}_{2, t}$ is given by

$$
\mathscr{G}_{2, t}:=\left(i \partial_{t} T_{t}\right) T_{t}^{*}+\mathscr{G}_{2, t}^{\mathscr{V}}+\mathscr{G}_{2, t}^{\mathscr{K}}+\mathscr{G}_{2, t}^{\lambda}
$$

with

$$
\begin{aligned}
\mathscr{G}_{2, t}^{\mathscr{V}}= & \mathfrak{b}_{0} \int \mathrm{d} x\left|\varphi_{t}(x)\right|^{2}\left[a^{*}\left(\mathrm{ch}_{x}\right) a\left(\mathrm{ch}_{x}\right)+a^{*}\left(\operatorname{sh}_{y}\right) a\left(\operatorname{sh}_{x}\right)\right. \\
& \left.+a^{*}\left(\mathrm{ch}_{x}\right) a^{*}\left(\operatorname{sh}_{x}\right)+a\left(\mathrm{ch}_{x}\right) a\left(\operatorname{sh}_{x}\right)\right] \\
& +\int \mathrm{d} x \mathrm{~d} y K_{1, t}(x ; y)\left[a^{*}\left(\mathrm{ch}_{x}\right) a\left(\mathrm{ch}_{y}\right)+a^{*}\left(\operatorname{sh}_{x}\right) a\left(\operatorname{sh}_{y}\right)\right. \\
& \left.+a^{*}\left(\operatorname{ch}_{x}\right) a^{*}\left(\operatorname{sh}_{y}\right)+a\left(\operatorname{ch}_{y}\right) a\left(\operatorname{sh}_{x}\right)\right] \\
& +\int \mathrm{d} x \mathrm{~d} y K_{2, t}(x ; y)\left[a^{*}\left(\operatorname{ch}_{x}\right) a\left(\operatorname{sh}_{y}\right)+a^{*}\left(\operatorname{ch}_{y}\right) a\left(\operatorname{sh}_{x}\right)\right. \\
& \left.+a^{*}\left(\operatorname{ch}_{x}\right) a^{*}\left(\operatorname{ch}_{y}\right)+a\left(\operatorname{sh}_{x}\right) a\left(\operatorname{sh}_{y}\right)+h . c .\right] \\
& +\frac{1}{2}\left[\left\|\varphi_{t}^{2}\right\|_{2}^{2} a^{*}\left(\varphi_{t}\right) a\left(\varphi_{t}\right)-2 a^{*}\left(\varphi_{t}\right) a\left(\left|\varphi_{t}\right|^{2} \varphi_{t}\right)+h . c .\right] \\
= & \sum_{i=1}^{4} \mathscr{G}_{2, t}^{\mathscr{V},(i)}
\end{aligned}
$$

and

$$
\mathscr{G}_{2, t}^{\lambda}=\frac{3 \mathfrak{b}_{0}}{8 \pi \ell^{3}} \int \mathrm{d} x \mathrm{~d} y \chi(|x-y| \leq \ell) \varphi_{t}^{2}((x+y) / 2) a_{x}^{*} a_{y}^{*}+h . c .
$$


and

$$
\begin{aligned}
\mathscr{G}_{2, t}^{\mathscr{K}}-\mathscr{K}= & \int \mathrm{d} x\left[a_{x}^{*} a\left(-\Delta_{x} \mathrm{p}_{x}\right)+a^{*}\left(-\Delta_{x} \mathrm{p}_{x}\right) a\left(\mathrm{ch}_{x}\right)+a^{*}\left(\mathrm{k}_{x}\right) a\left(-\Delta_{x} \mathrm{r}_{x}\right)\right. \\
& \left.+a^{*}\left(\nabla_{x} \mathrm{k}_{x}\right) a\left(\nabla_{x} \mathrm{k}_{x}\right)+a^{*}\left(-\Delta_{x} \mathrm{r}_{x}\right) a\left(\mathrm{r}_{x}\right)\right] \\
& +\int \mathrm{d} x\left[a_{x}^{*} a^{*}\left(-\Delta_{x} \mu_{x}\right)+a_{x}^{*} a^{*}\left(-\Delta_{x} \mathrm{r}_{x}\right)+a^{*}\left(-\Delta_{x} \mathrm{p}_{x}\right) a^{*}\left(\operatorname{sh}_{x}\right)\right. \\
& +a\left(-\Delta_{x} \mathrm{r}_{x}\right) a_{x}+a\left(-\Delta_{x} \mu_{x}\right) a_{x} \\
& \left.+a\left(\operatorname{sh}_{x}\right) a\left(-\Delta_{x} \mathrm{p}_{x}\right)+a^{*}\left(-\Delta_{x} \mathrm{r}_{x}\right) a\left(\mathrm{k}_{x}\right)\right] \\
& +\frac{1}{2} \int \mathrm{d} x \mathrm{~d} y \omega_{\infty}(x-y) \varphi_{t}((x+y) / 2) \Delta \varphi_{t}((x+y) / 2) a_{x}^{*} a_{y}^{*}+\text { h.c. } \\
& +\frac{1}{2} \int \mathrm{d} x \mathrm{~d} y \omega_{\infty}(x-y) \nabla \varphi_{t}((x+y) / 2) \cdot \nabla \varphi_{t}((x+y) / 2) a_{x}^{*} a_{y}^{*}+\text { h.c. }
\end{aligned}
$$

Here, we used the notation $\mathscr{K}=\int \mathrm{d} x a_{x}^{*}\left(-\Delta_{x}\right) a_{x}$ and $K_{1, t}=q_{t} \widetilde{K}_{1, t} q_{t}$ and $K_{2, t}=$ $\left(q_{t} \otimes q_{t}\right) \widetilde{K}_{2, t}$ where $\widetilde{K}_{1, t}$ is the operator with integral kernel

$$
\widetilde{K}_{1, t}(x, y)=\mathfrak{b}_{0} \varphi_{t}(x) \delta(x-y) \overline{\varphi_{t}(y)}
$$

and $K_{2, t}$ is the function given through

$$
\widetilde{K}_{2, t}(x, y)=\mathfrak{b}_{0} \varphi_{t}(x) \delta(x-y) \varphi_{t}(y) .
$$

Note that (1.19) implies $K_{1, t}, K_{2, t} \in L^{\infty}\left(\mathbb{R}^{6}\right) \cap L^{2}\left(\mathbb{R}^{6}\right)$ with norms uniform in $N$. Norm approximation. We consider the solution $\psi_{N, t}$ of the Schrödinger equation (1.2) with initial data $\psi_{N, 0}=U_{\varphi_{0}}^{*} \mathbb{1} \leq N T_{N, 0}^{*} \Omega$. It is proven in [11, Theorem 2] that for all $\alpha<\min \{\beta / 2,(1-\beta) / 2\}$ there exists a constant $C>0$ such that

$$
\left\|\mathscr{U}_{\varphi_{N, t}} \psi_{N, t}-e^{-i \int_{0}^{t} \mathrm{~d} \tau \eta_{N}(\tau)} T_{N, t}^{*} \mathscr{U}_{2}(t ; 0) \Omega\right\|^{2} \leq C N^{-\alpha} \exp (C \exp (C|t|))
$$

for all $N$ sufficiently large and all $t \in \mathbb{R}$.

\subsection{Bogoliubov transformation}

The limiting dynamics $\mathscr{U}_{2}(t ; s)$ defined in (1.26) is quadratic in creation and annihilation operators. As the following proposition shows, it gives rise to a Bogoliubov transformation defined in the following. For this, we first define

$$
A(f, g)=a^{*}(f)+a(\bar{g}) \text { for } f, g \in L^{2}\left(\mathbb{R}^{3}\right) .
$$


On the one hand,

$$
A^{*}(f, g)=A(\bar{g}, \bar{f})+A(\mathscr{J}(f, g)) \quad \text { with } \quad \mathscr{J}=\left(\begin{array}{ll}
0 & J \\
J & 0
\end{array}\right) \text {. }
$$

Here, $J: L^{2}\left(\mathbb{R}^{3}\right) \rightarrow L^{2}\left(\mathbb{R}^{3}\right)$ denotes the anti-linear operator defined by $J f=$ $\bar{f}$ for all $f \in L^{2}\left(\mathbb{R}^{3}\right)$. On the other hand, the commutation relations imply for $f_{1}, f_{2}, g_{1}, g_{2} \in L^{2}\left(\mathbb{R}^{3}\right)$

$$
\left[A\left(f_{1}, g_{1}\right), A^{*}\left(f_{2}, g_{2}\right)\right]=\left\langle\left(f_{1}, g_{1}\right), S\left(f_{2}, g_{2}\right)\right\rangle_{L^{2}\left(\mathbb{R}^{3}\right) \oplus L^{2}\left(\mathbb{R}^{3}\right)} \quad \text { with } \quad S=\left(\begin{array}{cc}
1 & 0 \\
0 & -1
\end{array}\right) .
$$

Definition 2 A Bogoliubov transformation is a linear map $v: L^{2}\left(\mathbb{R}^{3}\right) \oplus L^{2}\left(\mathbb{R}^{3}\right) \rightarrow$ $L^{2}\left(\mathbb{R}^{3}\right) \oplus L^{2}\left(\mathbb{R}^{3}\right)$ preserving the relations (1.33) and (1.34), i.e. $v^{*} S v=S$ and $\mathscr{J} v=v \mathscr{J}$.

It turns out that a Bogoliubov transformation $v$ is of the form

$$
v=\left(\begin{array}{ll}
U & J V J \\
V & J U J
\end{array}\right)
$$

for linear operators $U, V: L^{2}\left(\mathbb{R}^{3}\right) \rightarrow L^{2}\left(\mathbb{R}^{3}\right)$ satisfying $U^{*} U-V^{*} V=1$ and $U^{*} J V J-V^{*} J U J=0$.

The following proposition is proven in Sect. 2.2.

Proposition 1 Let $\mathscr{U}_{2}(t ; s)$ be the dynamics defined in (1.27). For every $t, s \in \mathbb{R}$, there exists a bounded linear map

$$
\Theta(t ; s)=L^{2}\left(\mathbb{R}^{3}\right) \oplus L^{2}\left(\mathbb{R}^{3}\right) \rightarrow L^{2}\left(\mathbb{R}^{3}\right) \oplus L^{2}\left(\mathbb{R}^{3}\right),
$$

such that

$$
\mathscr{U}_{2}^{*}(t ; s) A(f, g) \mathscr{U}_{2}(t ; s)=A(\Theta(t ; s)(f, g))
$$

for all $f, g \in L^{2}\left(\mathbb{R}^{3}\right)$. The map $\Theta(t ; s)$ satisfies

$$
\Theta(t ; s) \mathscr{J}=\mathscr{J} \Theta(t ; s), \quad S=\Theta(t ; s)^{*} S \Theta(t ; s),
$$

where $\mathscr{J}$ and $S$ are defined in (1.33) resp. (1.34). The Bogoliubov transformation $\Theta(t ; s)$ can be written as

$$
\Theta(t ; s)=\left(\begin{array}{ll}
U(t ; s) & J V(t ; s) J \\
V(t ; s) & J U(t ; s) J
\end{array}\right)
$$


for bounded linear maps $U(t ; s), V(t ; s): L^{2}\left(\mathbb{R}^{3}\right) \rightarrow L^{2}\left(\mathbb{R}^{3}\right)$ satisfying

$U^{*}(t ; s) U(t ; s)-V^{*}(t ; s) V(t ; s)=1, \quad U^{*}(t ; s) J V(t ; s) J=V^{*}(t ; s) J U(t ; s) J$.

\subsection{Central limit theorem}

From a probabilistic point of view, (1.7) implies a law of large numbers, in the sense that for a one-particle self-adjoint operator $O$ on $L^{2}\left(\mathbb{R}^{3}\right)$ and for every $\delta>0$

$$
\lim _{N \rightarrow \infty} \mathbb{P}_{\psi_{N, t}}\left(\left|\frac{1}{N} \sum_{j=1}^{N}\left(O^{(j)}-\left\langle\varphi_{t}, O \varphi_{t}\right\rangle\right)\right|>\delta\right)=0 .
$$

Here $O^{(j)}$ denotes the operator on $L^{2}\left(\mathbb{R}^{3 N}\right)$ acting as $O$ on the $j$ th particle and as identity elsewhere. The proof of (1.37) follows from Markov's inequality (see [13]). As a next step, we are interested in a central limit theorem. For this, we consider the rescaled random variable

$$
\mathscr{O}_{N, t}=\frac{1}{\sqrt{N}} \sum_{j=1}^{N}\left(O^{(j)}-\left\langle\varphi_{N, t}, O \varphi_{N, t}\right\rangle\right)
$$

where $\varphi_{N, t}$ denotes the solution of (1.12) with initial data $\varphi_{N, 0}=\varphi_{0}$.

We consider initial data $\psi_{N, 0}$ of the form $\psi_{N, 0}=\mathscr{U}_{\varphi_{0}}^{*} \mathbb{1} \leq N T_{N, 0}^{*} \Omega$ exhibiting BoseEinstein condensation [11, Theorem 3]. As a consequence, such a initial data satisfy a law of large numbers in the sense of (1.37). Moreover, such initial data obeys a central limit theorem in the sense that

$$
\mathbb{P}_{\psi_{N, 0}}\left(\mathscr{O}_{N, 0} \in[a ; b]\right) \rightarrow \mathbb{P}\left(G_{0} \in[a, b]\right) \quad \text { as } \quad N \rightarrow \infty
$$

for every $-\infty<a<b<\infty$. Here, $G_{0}$ denotes the centered Gaussian random variable with variance $\left\|\sigma_{0}\right\|_{2}^{2}$, where

$$
\sigma_{0}=\sinh _{\eta_{0}} \overline{q_{0} O \varphi_{0}}+\cosh _{\eta_{0}} q_{0} O \varphi_{0}
$$

following from Theorem 1 for time $t=0$.

Note that initial data of the form $\psi_{N, 0}=\mathscr{U}_{\varphi_{0}}^{*} \mathbb{1} \leq N T_{N, 0}^{*} \Omega$ describe approximate ground states of trapped systems [9]. In experiments, such initial data are prepared by trapping particles through external fields and by cooling them down to extremely low temperatures so that the system essentially relaxes to its ground state.

The validity of a central limit theorem for the ground state of trapped systems has already been addressed in [32]. To be more precise, [32] considers the ground state of (1.1) for $\beta=1$, i.e. in the Gross-Pitaevskii regime. The ground state is known to exhibit Bose-Einstein condensation. It is proven that the ground state satisfies a 
central limit theorem. The arguments of the proof can be adapted to the intermediate regime $\beta<1$ using the norm approximation for the ground state obtained in [9].

Now, we consider the time evolution of the initial data $\psi_{N, 0}=\mathscr{U}_{\varphi_{0}}^{*} \mathbb{1} \leq N T_{N, 0}^{*} \Omega$ with respect to the Schrödinger equation (1.2) and show the validity of a (multi-variate) central limit theorem.

Theorem 1 Let $\beta \in(0,1)$ and assume $V$ to be radially symmetric, smooth, compactly supported and point-wise nonnegative. Furthermore, fix $\ell>0$ (independent of $N$ ). Let $\varphi_{t}$ denote the solution of (1.3) and $\varphi_{N, t}$ the solution of (1.12) both with initial data $\varphi_{0} \in H^{4}\left(\mathbb{R}^{3}\right)$. Moreover, we denote by $\psi_{N, t}$ the solution of the Schrödinger equation (1.2) with initial data $\psi_{N, 0}=\mathscr{U}_{\varphi_{N, 0}^{*}}^{*} \mathbb{1}^{\leq N} T_{N, 0}^{*} \Omega$ (where $\mathscr{U}_{\varphi_{N, 0}}$ and $T_{N, 0}$ are defined in (1.8) resp. (1.13)). For $k \in \mathbb{N}$, let $O_{1}, \ldots, O_{k}$ be bounded operators on $L^{2}\left(\mathbb{R}^{3}\right)$. We define $v_{j, t} \in L^{2}\left(\mathbb{R}^{3}\right)$ through

$$
\begin{aligned}
v_{j, t}= & \left(U(t ; 0) \cosh _{\eta_{t}}+\bar{V}(t ; 0) \sinh _{\eta_{t}}\right) q_{t} O_{j} \varphi_{t} \\
& +\left(U(t ; 0) \sinh _{\eta_{t}}+\bar{V}(t ; 0) \cosh _{\eta_{t}}\right) \overline{q_{t} O_{j} \varphi_{t}}
\end{aligned}
$$

where the operators $U(t ; 0), V(t ; 0) \in L^{2}\left(\mathbb{R}^{3} \times \mathbb{R}^{3}\right)$ are defined in Proposition 1 , $q_{t}=1-\left|\varphi_{t}\right\rangle\left\langle\varphi_{t}\right|$ and $\eta_{t}$ as defined in (1.20).

Assume $\Sigma_{t} \in \mathbb{C}^{k \times k}$, given through

$$
\left(\Sigma_{t}\right)_{i, j}= \begin{cases}\left\langle v_{i, t}, v_{j, t}\right\rangle & \text { for } i<j \\ \left\langle v_{j, t}, v_{i, t}\right\rangle & \text { otherwise }\end{cases}
$$

is invertible.

Furthermore, let $g_{1}, \ldots g_{k} \in L^{1}(\mathbb{R})$ with $\widehat{g}_{i} \in L^{1}\left(\mathbb{R},(1+|s|)^{4} d s\right)$ for all $i \in$ $\{1, \ldots k\}$ and let $\mathscr{O}_{j, N, t}$ denote the random variable (1.38) associated with $O_{j}$ for all $j \in\{1, \ldots, k\}$. For every $\alpha<\min \{\beta / 2,(1-\beta) / 2\}$, there exists $C>0$ such that

$$
\begin{aligned}
& \mid \mathbb{E}_{\psi_{N, t}}\left[g_{1}\left(\mathscr{O}_{1, N, t}\right) \ldots g_{k}\left(\mathscr{O}_{k, N, t}\right)\right] \\
& \quad-\frac{1}{\sqrt{(2 \pi)^{k} \operatorname{det} \Sigma}} \int d x_{1} \ldots d x_{k} g_{1}\left(x_{1}\right) \ldots g_{k}\left(x_{k}\right) e^{-\frac{1}{2} \sum_{i, j=1}^{k} \Sigma_{i, j}^{-1} x_{j} x_{j}} \mid \\
& \quad \leq C \exp (\exp (C|t|)) N^{-\alpha} \prod_{j=1}^{k} \int d \tau\left|\widehat{g}_{j}(\tau)\right|\left(1+N^{\alpha-\gamma}|\tau|^{2}+N^{\alpha-1 / 2}|\tau|^{3}+N^{\alpha-1}|\tau|^{4}\right),
\end{aligned}
$$

where $\gamma=\min \{\beta,(1-\beta)\}$.

A similar result has been established in $[6,13]$ for the mean-field regime characterized through weak interaction of the particles. It is shown that fluctuations around the nonlinear Hartree equation of bounded self-adjoint one-particle operators satisfy a (multi-variate) central limit theorem. We show that this result is true in the intermediate regime, where the interaction is singular, too. In particular, the correlation structure which becomes of importance in the intermediate regime does not affect the 
validity of a central limit theorem. However, it affects the covariance matrix (1.41) through the Bogolioubiv transform $T_{t}$.

Similarly as in [13, Corollary 1.3 ], Theorem 1 implies a Berry-Esséen-type central limit theorem. To be more precise, we consider a bounded self-adjoint operator $O$ on $L^{2}\left(\mathbb{R}^{3}\right)$ and the random variable

$$
\mathscr{O}_{N, t}=\frac{1}{\sqrt{N}} \sum_{i=1}^{N}\left(O^{(i)}-\left\langle\varphi_{N, t}, O \varphi_{N, t}\right\rangle\right) .
$$

For every $\alpha<\min \{\beta / 2,(1-\beta) / 2\}$ and $-\infty<a<b<\infty$, there exists a constant $C>0$ such that

$$
\left|\mathbb{P}_{\psi_{N, t}}\left(\mathscr{O}_{N, t} \in[a ; b]\right)-\mathbb{P}\left(G_{t} \in[a ; b]\right)\right| \leq C N^{-\alpha / 2},
$$

where $G_{t}$ is the centered Gaussian random variable with variance $\left\|\sigma_{t}\right\|_{2}^{2}$ and $\sigma_{t} \in$ $L^{2}\left(\mathbb{R}^{3}\right)$ is defined through

$$
\sigma_{t}=\left(U(t ; 0) \cosh _{\eta_{t}}+\bar{V}(t ; 0) \sinh _{\eta_{t}}\right) q_{t} O \varphi_{t}+\left(U(t ; 0) \sinh _{\eta_{t}}+\bar{V}(t ; 0) \cosh _{\eta_{t}}\right) \overline{q_{t} O \varphi_{t}} .
$$

Note that Theorem 1 resp. (1.42) implies that fluctuations around the nonlinear Hartree equation with singular interaction satisfy a (multi-variate) central limit theorem. Comparing with $\sigma_{0}$ from (1.40), the fluctuations enter in the variance $\sigma_{t}$ through the operators $U(t ; 0), V(t ; 0)$ as defined in Proposition (1) and the Bogoliubov transformation (1.21).

Moreover, note that the covariance matrix (1.41) resp. the variance (1.43) are completely determined by the Bogoliubov transform $T_{t}$ defined in (1.21) and the quadratic fluctuation dynamics $\mathscr{U}_{2}(t ; 0)$ defined in (1.27). Theorem 1 resp. the properties (1.36) of the operators $U(t ; 0), V(t ; 0)$ show that the solution of the Schrödinger equation (1.2) modulo the extraction of the condensate is approximately a quasi-free state for quasi-free initial data. This observation coincides with results in [24,28,29].

\section{Proof of results}

\subsection{Preliminaries}

The proof of Theorem 1 is based on the norm approximation (1.31) from [11]. In the following, we collect useful properties of the unitaries used therein.

To this end, we define the more general quadratic dynamics $\mathscr{U}_{\text {gen }}(t ; s)$.

Definition 3 Let $\mathscr{U}_{\text {gen }}(t ; s)$ be the dynamics satisfying

$$
i \partial_{t} \mathscr{U}_{\text {gen }}(t ; s)=\mathscr{G}_{\text {gen }, t} \mathscr{U}_{\text {gen }}(t ; s)
$$


where the generator $\mathscr{G}_{\mathrm{gen}, t}$ is of the form

$$
\begin{aligned}
\mathscr{G}_{\mathrm{gen}, t}= & \int \mathrm{d} x \nabla_{x} a_{x}^{*} \nabla_{x} a_{x}+\int \mathrm{d} x \mathrm{~d} y H_{t}^{(1)}(x ; y) a_{x}^{*} a_{y} \\
& +\int \mathrm{d} x \mathrm{~d} y\left(H_{t}^{(2)}(x, y) a_{x}^{*} a_{y}^{*}+\overline{H_{t}^{(2)}}(x, y) a_{x} a_{y}\right)+c
\end{aligned}
$$

with

$$
\left\|H_{t}^{(1)}\right\| \leq C e^{C|t|}, \quad\left\|H_{t}^{(2)}\right\|_{2} \leq C e^{C|t|}
$$

for constants $c, C>0$.

In the following, we prove the results for the dynamics $\mathscr{U}_{\text {gen }}(t ; s)$. As the next Lemma shows, the results then apply to $\mathscr{U}_{2}(t ; s)$, too.

Lemma 1 The dynamics $\mathscr{U}_{2}(t ; s)$ defined in Definition 1 is of the form of $\mathscr{U}_{\mathrm{gen}}(t ; s)$ defined in Definition 3.

Proof By the definition (1.27) of $\mathscr{G}_{2, t}$, we split

$$
\mathscr{G}_{2, t}-\mathscr{K}=\left(i \partial_{t} T_{t}\right) T_{t}^{*}+\mathscr{G}_{2, t}^{\mathscr{V}}+\left(\mathscr{G}_{2, t}^{\mathscr{K}}-\mathscr{K}\right)+\mathscr{G}_{2, t}^{\lambda}
$$

and consider each of the summands separately. First, we consider $\mathscr{G}_{2, t}^{\mathscr{V}}$ defined in (1.28), which is again split into four terms. The first one, $\mathscr{G}_{2, t}^{\mathscr{V}, 1}$ of the r.h.s. of (1.28), satisfies assumption (2.3) since on the one hand

$$
\left\|\operatorname{ch}_{\eta_{t}}\left|\varphi_{t}\right|^{2} \operatorname{ch}_{\eta_{t}}\right\|_{2} \leq\left\|\varphi_{t}\right\|_{4}^{2} \leq C e^{C|t|}, \quad\left\|\left.\operatorname{sh}_{\eta_{t}} \varphi_{t}\right|^{2} \operatorname{sh}_{\eta_{t}}\right\|_{2} \leq\left\|\varphi_{t}\right\|_{\infty}^{2}\left\|\operatorname{sh}_{\eta_{t}}\right\|_{2}^{2} \leq C e^{C|t|}
$$

and

$$
\left\|\operatorname{ch}_{\eta_{t}}\left|\varphi_{t}\right|^{2} \operatorname{sh}_{\eta_{t}}\right\|_{2} \leq C\left\|\varphi_{t}\right\|_{2}\left\|\varphi_{t}\right\|_{\infty}\left\|\operatorname{sh}_{\eta_{t}}\right\|_{2} \leq C e^{C|t|}
$$

following from (1.19) and (1.23). For the same reasons, the second term $\mathscr{G}_{2, t}^{\mathscr{V}, 2}$ of the r.h.s. of (1.28) satisfies assumption (2.3), too. For the third term $\mathscr{G}_{2, t}^{\mathscr{V}, 3}$, the definition of $K_{2, t}$ implies

$$
\begin{aligned}
\left\|K_{2, t} \operatorname{sh}_{\eta_{t}}\right\|_{2} & \leq\left\|\varphi_{t}\right\|_{4}^{1 / 2}\left\|\operatorname{sh}_{\eta_{t}}\right\|_{2} \leq C e^{C|t|} \\
\left\|\mathrm{p}_{t} K_{2, t} \operatorname{sh}_{\eta_{t}}\right\| & \leq\left\|\operatorname{sh}_{\eta_{t}}\right\|_{2}\left\|\mathrm{p}_{t}\right\|_{2}\left\|\varphi_{t}\right\|_{4}^{1 / 2} \leq C e^{C|t|}
\end{aligned}
$$

again from (1.19) and (1.23). The fourth term $\mathscr{G}_{2, t}^{\mathscr{V}, 4}$ satisfies the assumption (2.2) due to (1.19). 
Furthermore, a transformation of variables shows

$$
\begin{aligned}
& \left.\int \mathrm{d} x \mathrm{~d} y \chi(|x-y| \leq \ell) \mid \varphi_{t}((x+y) / 2)\right)\left.\right|^{4} \\
& \quad=\int \mathrm{d} x \mathrm{~d} y \chi(|x| \leq \ell)\left|\varphi_{t}(y)\right|^{4}=C\left\|\varphi_{t}\right\|_{4}^{4} \leq C e^{C|t|} .
\end{aligned}
$$

Therefore, $\mathscr{G}_{2, t}^{\lambda}$ is of form (2.2).

Moreover, for the term $\mathscr{G}_{2, t}^{\mathscr{K}}-\mathscr{K}$, we observe with (1.23) and (1.25)

$$
\left\|\Delta_{1} p_{t}\right\|_{2} \leq C e^{C|t|},\left\|\Delta_{1} \mu_{t}\right\|_{2} \leq C e^{C|t|},\left\|\Delta_{1} \mathrm{r}_{t}\right\|_{2} \leq C e^{C|t|} .
$$

The remaining bounds follow in the same way. Note that (1.24) implies the bound

$$
\left\|\nabla_{1} k_{t} \nabla_{1} k_{t}\right\| \leq \max \left\{\sup _{x} \int \mathrm{d} z\left|\nabla_{1} k(x ; z)\right|, \sup _{y} \int \mathrm{d} z\left|\nabla_{1} k(z ; y)\right|\right\} \leq C \text {. }
$$

Moreover, by definition (1.17) of the limiting kernel, $\omega_{\infty} \in L^{p}\left(\mathbb{R}^{3}\right)$ for all $p<3$. Hence, the remaining terms of $\mathscr{G}_{2, t}^{\mathscr{K}}$ satisfy the assumptions, too.

We are left with the first term of the r.h.s. of (2.4). We write $T_{t}=e^{-B\left(\eta_{t}\right)}$. The properties (1.14) of the Bogoliubov transformation lead to

$$
\begin{aligned}
\left(\partial_{t} T_{t}\right) T_{t}^{*}= & -\int_{0}^{1} \mathrm{~d} s e^{-s B\left(\eta_{t}\right)}\left(\partial_{t} B\left(\eta_{t}\right)\right) e^{s B\left(\eta_{t}\right)} \\
= & \int_{0}^{1} \mathrm{~d} s \int \mathrm{d} x \mathrm{~d} y e^{-s B\left(\eta_{t}\right)}\left(\dot{\eta}_{t}(x ; y) a_{x}^{*} a_{y}^{*}+h . c .\right) e^{s B\left(\eta_{t}\right)} \\
= & \int \mathrm{d} x \mathrm{~d} y \dot{\eta}_{t}(x ; y)\left(a^{*}\left(\operatorname{ch}_{x}\right) a^{*}\left(\operatorname{ch}_{y}\right)+a\left(\operatorname{sh}_{x}\right) a\left(\operatorname{sh}_{y}\right)\right)+\text { h.c. } \\
& +\int \mathrm{d} x \mathrm{~d} y \dot{\eta}_{t}(x ; y)\left(a^{*}\left(\operatorname{ch}_{x}\right) a\left(\operatorname{sh}_{y}\right)+a^{*}\left(\operatorname{ch}_{y}\right) a\left(\operatorname{sh}_{x}\right)\right)+\text { h.c. } \\
& +\int \mathrm{d} x \mathrm{~d} y \dot{\eta}_{t}(x ; y) \operatorname{sh}_{x} \operatorname{ch}_{y} .
\end{aligned}
$$

Since $\left\|\dot{\eta}_{t}\right\|_{2} \leq C e^{C|t|}$ from (1.24), these terms satisfy assumption (2.3), too.

As proven in [11, Proposition 8], any moments of the number of particles operator are approximately preserved with respect to conjugation with the Bogoliubov transformation $T_{N, t}$. To be more precise for every fixed $k \in \mathbb{N}$ and $\delta>0$, there exists $C>0$ such that

$$
\pm\left(T_{N, t} \mathscr{N}^{k} T_{N, t}^{*}-\mathscr{N}^{k}\right) \leq \delta \mathscr{N}^{k}+C
$$

As the following Lemma shows, the moments of number of particles operator are propagated in time with respect to the quadratic $\mathscr{U}_{\text {gen }}(t ; 0)$. 
Lemma 2 Let $\mathscr{U}_{\mathrm{gen}}(t ; s)$ be as defined in Definition 3 and $\psi \in \mathscr{F}$. For every $k \in \mathbb{N}$, there exists a constant $C>0$ such that for all $t \in \mathbb{R}$

$\left\langle\psi, \mathscr{U}_{\mathrm{gen}}(t ; s)^{*}(\mathscr{N}+1)^{k} \mathscr{U}_{\mathrm{gen}}(t ; s) \psi\right\rangle \leq C \exp (C \exp (C|t-s|))\left\langle\psi,(\mathscr{N}+1)^{k} \psi\right\rangle$

Proof We compute the derivative

$$
\begin{aligned}
i \frac{d}{d t} & \left\langle\psi, \mathscr{U}_{\mathrm{gen}}^{*}(t ; s)(\mathscr{N}+1)^{k} \mathscr{U}_{\mathrm{gen}}(t ; s) \psi\right\rangle \\
& =\left\langle\psi, \mathscr{U}_{\mathrm{gen}}^{*}(t ; s)\left[\mathscr{G}_{\mathrm{gen}, t},(\mathscr{N}+1)^{k}\right] \mathscr{U}_{\mathrm{gen}}(t ; s) \psi\right\rangle \\
& =\sum_{i=1}^{k}\left\langle\psi, \mathscr{U}_{\mathrm{gen}}^{*}(t ; s)(\mathscr{N}+1)^{i-1}\left[\mathscr{G}_{\mathrm{gen}, t}, \mathscr{N}\right](\mathscr{N}+1)^{k-i} \mathscr{U}_{\mathrm{gen}}(t ; s) \psi\right\rangle .
\end{aligned}
$$

Using the commutation relations and the definition (2.2), we find

$$
\begin{aligned}
i \frac{d}{d t}\left\langle\psi, \mathscr{U}_{\mathrm{gen}}^{*}(t ; s)(\mathscr{N}+1)^{k} \mathscr{U}_{\mathrm{gen}}(t ; s) \psi\right\rangle \\
=2 \sum_{i=1}^{k} \int \mathrm{d} x \mathrm{~d} y H_{t}^{(2)}(x, y)\left\langle\psi, \mathscr{U}_{\mathrm{gen}}^{*}(t ; s)(\mathscr{N}+1)^{i-1} a_{x}^{*} a_{y}^{*}(\mathscr{N}+1)^{k-i} \mathscr{U}_{\mathrm{gen}}(t ; s) \psi\right\rangle \\
\quad+2 \sum_{i=1}^{k} \int \mathrm{d} x \mathrm{~d} y \overline{H_{t}^{(2)}(x, y)}\left\langle\psi, \mathscr{U}_{\mathrm{gen}}^{*}(t ; s)(\mathscr{N}+1)^{i-1} a_{x} a_{y}(\mathscr{N}+1)^{k-i} \mathscr{U}_{\mathrm{gen}}(t ; s) \psi\right\rangle .
\end{aligned}
$$

For the first term of the right-hand side, the commutation relations yield

$$
\begin{aligned}
& \left|\int \mathrm{d} x \mathrm{~d} y H_{t}^{(2)}(x ; y)\left\langle\psi, \mathscr{U}_{\mathrm{gen}}^{*}(t ; s)(\mathscr{N}+1)^{i-1} a_{x}^{*} a_{y}^{*}(\mathscr{N}+1)^{k-i} \mathscr{U}_{\mathrm{gen}}(t ; s) \psi\right\rangle\right| \\
& \quad \leq \int \mathrm{d} x \mathrm{~d} y\left|H_{t}^{(2)}(x ; y)\right|\left\|(\mathscr{N}+1)^{(k+1) / 2-i} a_{x}^{*}(\mathscr{N}+1)^{i-1} \mathscr{U}_{\mathrm{gen}}(t ; s) \psi\right\| \\
& \quad \times\left\|(\mathscr{N}+1)^{i-(k-1) / 2} a_{y}(\mathscr{N}+1)^{k-i} \mathscr{U}_{\mathrm{gen}}(t ; s) \psi\right\| \\
& \quad \leq C\left\|H_{t}^{(2)}\right\|_{2}\left\|(\mathscr{N}+1)^{k / 2} \mathscr{U}_{\mathrm{gen}}(t ; s) \psi\right\|^{2} \leq C e^{C|t|}\left\langle\psi, \mathscr{U}_{\mathrm{gen}}^{*}(t ; s)(\mathscr{N}+1)^{k} \mathscr{U}_{\mathrm{gen}}(t ; s) \psi\right\rangle,
\end{aligned}
$$

where $C$ depends on $k \in \mathbb{N}$. The second of the r.h.s. of (2.6) follows in the same way. Hence, there exists $C>0$ such that

$$
\left|\frac{d}{d t}\left\langle\psi, \mathscr{U}_{\mathrm{gen}}^{*}(t ; s)(\mathscr{N}+1)^{k} \mathscr{U}_{\mathrm{gen}}(t ; s) \psi\right\rangle\right| \leq C e^{C|t|}\left\langle\psi, \mathscr{U}_{\mathrm{gen}}^{*}(t ; s)(\mathscr{N}+1)^{k} \mathscr{U}_{\mathrm{gen}}(t ; s) \psi\right\rangle .
$$

Hence, the Gronwall inequality implies

$$
\left\langle\psi, \mathscr{U}_{\mathrm{gen}}^{*}(t ; s)(\mathscr{N}+1)^{k} \mathscr{U}_{\mathrm{gen}}(t ; s) \psi\right\rangle \leq C \exp (C \exp (C|t-s|))\left\langle\psi,(\mathscr{N}+1)^{k} \psi\right\rangle .
$$


For $f \in L^{2}\left(\mathbb{R}^{3}\right)$, let $\phi_{a}(f)=a^{*}(f)+a(f)$. In [13, Proposition 3.4], it is shown that for every $k \in \mathbb{N}$ and $\delta \in \mathbb{R}$, there exists a constant $C>0$ such that

$$
\left\langle\psi, e^{-i s\left(\phi_{a}(f)+\delta \mathrm{d} \Gamma(H)\right)}(\mathscr{N}+1)^{k} e^{i s\left(\phi_{a}(f)+\delta \mathrm{d} \Gamma(H)\right)} \psi\right\rangle \leq C\left\langle\psi,\left(\mathscr{N}+\alpha+s^{2}\|f\|^{2}\right)^{k} \psi\right\rangle
$$

for all $\psi \in \mathscr{F}$ and $\alpha \geq 1$. Hereafter, we denote $\mathrm{d} \Gamma(H)=\sum_{j=1}^{N} H^{(j)}$ for a bounded operator $H$ on $L^{2}\left(\mathbb{R}^{3}\right)$. A similar estimate holds true for when replacing the creation and annihilation operators $a(f), a^{*}(f)$ with the modified ones $b^{*}(f), b(f)$ defined in (1.9). Let $\phi_{b}(f)=b^{*}(f)+b(f)$. In fact, as proven in [32, Lemma 3.2], for every $k \in \mathbb{N}$, there exists a constant $C>0$ such that

$$
\left\langle\xi, e^{-i \phi_{b}(h)}\left(\mathscr{N}_{+}(t)+1\right)^{k} e^{i \phi_{b}(h)} \xi\right\rangle \leq C\left\langle\xi,\left(\mathscr{N}_{+}(t)+\alpha+\|f\|^{2}\right)^{k} \xi\right\rangle
$$

for all $\xi \in \mathscr{F}_{+}^{\leq N}(t)$ and $\alpha \geq 1$.

\subsection{Proof of Proposition 1}

It follows from Lemma 1 that it is enough to prove Proposition 1 with respect the dynamics $\mathscr{U}_{\text {gen }}(t ; s)$.

First, we prove that for $f \in L^{2}\left(\mathbb{R}^{3}\right)$ the Fock space vectors $\mathscr{U}_{\text {gen }}^{*}(t ; s) a^{*}(f) \mathscr{U}_{\text {gen }}$ $(t ; s) \Omega$ and $\mathscr{U}_{\text {gen }}^{*}(t ; s) a(f) \mathscr{U}_{\text {gen }}(t ; s) \Omega$ are elements of the one-particle sector. The following Lemma is a generalization of [14, Lemma 8.1].

Lemma 3 Let $\mathscr{U}_{\mathrm{gen}}(t ; s)$ be the dynamics defined Definition 3. Then for all $f \in L^{2}(\mathbb{R})$,

$$
\mathscr{U}_{\mathrm{gen}}^{*}(t ; s) a^{\sharp}(f) \mathscr{U}_{\mathrm{gen}}(t ; s) \Omega=\mathscr{P}_{1} \mathscr{U}_{\mathrm{gen}}^{*}(t ; s) a^{\sharp}(f) \mathscr{U}_{\mathrm{gen}}(t ; s) \Omega,
$$

where either $a^{\sharp}(f)=a(f)$ or $a^{\sharp}(f)=a^{*}(f)$ and where $\mathscr{P}_{1}$ denotes the projection onto the one-particle sector of the Fock space $\mathscr{F}$.

Proof The proof follows the arguments of the proof of [14, Lemma 8.1]. For $m \in \mathbb{N}$, $m \neq 1$, we define for arbitrary $m$-particle wave function $\psi \in \mathscr{F}$ with $\|\psi\|=1$ the function

$$
\begin{aligned}
F(t)= & \sup _{\|f\|_{2} \leq 1}\left|\left\langle\psi, \mathscr{U}_{\mathrm{gen}}^{*}(t ; s) a(f) \mathscr{U}_{\mathrm{gen}}(t ; s) \Omega\right\rangle\right| \\
& +\sup _{\|f\|_{2} \leq 1}\left|\left\langle\psi, \mathscr{U}_{\mathrm{gen}}^{*}(t ; s) a^{*}(f) \mathscr{U}_{\mathrm{gen}}(t ; s) \Omega\right\rangle\right|
\end{aligned}
$$

Since $m \neq 1$, we observe that $F(s)=0$ and furthermore

$$
e^{i \mathscr{K} t} a(f) e^{-i \mathscr{K} t}=a\left(e^{-i \Delta t} f\right)=a\left(f_{t}\right)
$$


using the notation $f_{t}=e^{-i t \Delta} f$. Since $e^{-i \Delta t}$ is a unitary operator, we find

$$
\begin{aligned}
F(t)= & \sup _{\|f\|_{2} \leq 1}\left|\left\langle\psi, \mathscr{U}_{\text {gen }}^{*}(t ; s) e^{i \mathscr{K} t} a(f) e^{-i \mathscr{K} t} \mathscr{U}_{\operatorname{gen}}(t ; s) \Omega\right\rangle\right| \\
& +\sup _{\|f\|_{2} \leq 1}\left|\left\langle\psi, \mathscr{U}_{\text {gen }}^{*}(t ; s) e^{i \mathscr{K} t} a^{*}(f) e^{-i \mathscr{K} t} \mathscr{U}_{\operatorname{gen}}(t ; s) \Omega\right\rangle\right| .
\end{aligned}
$$

Then,

$$
\begin{aligned}
& i \frac{d}{d t}\left\langle\psi, \mathscr{U}_{\mathrm{gen}}^{*}(t ; s) e^{i \mathscr{K} t} a(f) e^{-i \mathscr{K} t} \mathscr{U}_{\mathrm{gen}}(t ; s) \Omega\right\rangle \\
& \quad=\left\langle\psi, \mathscr{U}_{\mathrm{gen}}^{*}(t ; s)\left[a\left(f_{t}\right), \mathscr{G}_{\mathrm{gen}, t}-\mathscr{K}\right] \mathscr{U}_{\mathrm{gen}}(t ; s) \Omega\right\rangle
\end{aligned}
$$

and the definition of $\mathscr{G}_{\mathrm{gen}, t}$ in (2.2) leads to

$$
\begin{aligned}
{\left[a\left(f_{t}\right), \mathscr{G}_{\mathrm{gen}, t}-\mathscr{K}\right]=} & \int \mathrm{d} x \mathrm{~d} y\left(f_{t}(x) H_{t}^{(1)}(x ; y)\right) a_{y} \\
& +\int \mathrm{d} x \mathrm{~d} y\left(H_{t}^{(2)}(x ; y) f_{t}(x)+H_{t}^{(2)}(y ; x) f_{t}(x)\right) a_{y}^{*}
\end{aligned}
$$

The assumption (2.3) implies on the one hand

$$
\left\|H_{t}^{(1)} f_{t}\right\|_{2} \leq C e^{C|t|}\left\|f_{t}\right\|_{2}
$$

and on the other hand

$$
\left\|H_{t}^{(2)} f_{t}\right\|_{2} \leq\left\|f_{t}\right\|_{2}\left\|H_{t}^{(2)}\right\|_{2} \leq C e^{C|t|}\left\|f_{t}\right\|_{2} .
$$

Hence,

$$
\left|\left\langle\psi, \mathscr{U}_{\text {gen }}^{*}(t ; s) e^{i \mathscr{K} t} a(f) e^{-i \mathscr{K} t} \mathscr{U}_{\text {gen }}(t ; s) \Omega\right\rangle\right| \leq C \int_{0}^{t} \mathrm{~d} \tau e^{C|\tau|} F(\tau),
$$

and analogously,

$$
\left|\left\langle\psi, \mathscr{U}_{\text {gen }}^{*}(t ; s) e^{i \mathscr{K} t} a^{*}(f) e^{-i \mathscr{K} t} \mathscr{U}_{\operatorname{gen}}(t ; s) \Omega\right\rangle\right| \leq C \int_{0}^{t} \mathrm{~d} \tau e^{C|\tau|} F(\tau) .
$$

Note that these bounds are independent of $f \in L^{2}\left(\mathbb{R}^{3}\right)$. Thus,

$$
0 \leq F(t) \leq C \int_{0}^{t} \mathrm{~d} \tau e^{C|\tau|} F(\tau)
$$

Using the bounds $\left\|a^{\sharp}(f) \psi\right\| \leq\|f\|_{2}\left\|(\mathscr{N}+1)^{1 / 2} \psi\right\|$, we obtain

$$
F(t) \leq 2\left\|(\mathscr{N}+1)^{1 / 2} \mathscr{U}_{\mathrm{gen}}(t ; s) \Omega\right\| \leq C \exp (\exp (C|t-s|))\langle\psi,(\mathscr{N}+1) \psi\rangle
$$


Here, we used Lemma 2 for the last estimate. Since $F(s)=0$, the Gronwall inequality implies $F(t)=0$ for all $t \in \mathbb{R}$.

Proof of Proposition 1 We prove the Proposition with respect to the dynamics $\mathscr{U}_{\text {gen }}(t ; s)$ defined in Definition 3. Then, Proposition 1 follows from Lemma 1.

The proof follows the arguments of the proof of [6, Theorem 2.2]. Let $\mathscr{P}_{k}$ denote the projection onto the $k$-particle sector $\mathscr{F}_{k}$ of the Fock space. It follows from Lemma that 3

$$
\mathscr{P}_{k} \mathscr{U}_{\mathrm{gen}}^{*}(t ; s) a^{*}(f) \mathscr{U}_{\mathrm{gen}}(t ; s) \Omega=0, \quad \mathscr{P}_{k} \mathscr{U}_{\mathrm{gen}}^{*}(t ; s) a^{*}(f) \mathscr{U}_{\mathrm{gen}}(t ; s) \Omega=0
$$

for all $f \in L^{2}\left(\mathbb{R}^{3}\right)$ and $k \neq 1$. Thus, there exist linear operators $U(t ; s), V(t ; s)$ : $L^{2}\left(\mathbb{R}^{3}\right) \rightarrow L^{2}\left(\mathbb{R}^{3}\right)$ such that

$$
\begin{aligned}
& \mathscr{U}_{\mathrm{gen}}^{*}(t ; s) a^{*}(f) \mathscr{U}_{\mathrm{gen}}(t ; s) \Omega=a^{*}(U(t ; s) f) \Omega, \\
& \mathscr{U}_{\mathrm{gen}}^{*}(t ; s) a^{*}(f) \mathscr{U}_{\mathrm{gen}}(t ; s) \Omega=a^{*}(J V(t ; s) f) \Omega,
\end{aligned}
$$

where $J: L^{2}\left(\mathbb{R}^{3}\right) \rightarrow L^{2}\left(\mathbb{R}^{3}\right)$ denotes the anti-linear operator defined by $J f=\bar{f}$ for all $f \in L^{2}\left(\mathbb{R}^{3}\right)$. The operators $U(t ; s)$ and $V(t ; s)$ are bounded in $L^{2}\left(\mathbb{R}^{3}\right)$. This follows from Lemma 2 , since

$$
\begin{aligned}
\|U(t ; s) f\| & =\left\|a^{*}(U(t ; s) f) \Omega\right\|=\left\|a^{*}(f) \mathscr{U}_{\mathrm{gen}}(t ; s) \Omega\right\| \\
& \leq\|f\|\left\|(\mathscr{N}+1)^{1 / 2} \mathscr{U}_{\mathrm{gen}}(t ; s) \Omega\right\| \leq C \exp (c|t|)
\end{aligned}
$$

and

$$
\begin{aligned}
\|V(t ; s) f\| & =\left\|a^{*}(J V(t ; s) f) \Omega\right\|=\left\|a(f) \mathscr{U}_{\mathrm{gen}}(t ; s) \Omega\right\| \\
& \leq\|f\|\left\|\mathscr{N}^{1 / 2} \mathscr{U}_{\mathrm{gen}}(t ; s) \Omega\right\| \leq C e^{K|t|} .
\end{aligned}
$$

We define the bounded operator $\Theta$ on $L^{2}\left(\mathbb{R}^{3}\right) \oplus L^{2}\left(\mathbb{R}^{3}\right)$ through

$$
\Theta(t ; s)=\left(\begin{array}{ll}
U(t ; s) & J V(t ; s) J \\
V(t ; s) & J U(t ; s) J
\end{array}\right) .
$$

Then

$$
\mathscr{U}_{\text {gen }}^{*}(t ; s) A(f, g) \mathscr{U}_{\text {gen }}(t ; s) \Omega=A(\Theta(t ; s)(f, g)) \Omega
$$

for all $f, g \in L^{2}\left(\mathbb{R}^{3}\right)$. For fixed $\psi \in \mathscr{D}(\mathscr{K}+\mathscr{N}), g \in L^{2}\left(\mathbb{R}^{3}\right), s \in \mathbb{R}$ and any bounded operator $\mathscr{M}$ on $\mathscr{F}$ with $\mathscr{M} \mathscr{D}(\mathscr{K}+\mathscr{N}) \subset \mathscr{D}(\mathscr{K}+\mathscr{N})$, we define furthermore

$$
F(t)=\sum_{\sharp} \sup _{\|f\|_{2} \leq 1}\left\|\left[\left[\mathscr{U}_{\mathrm{gen}}^{*}(t ; s) a^{\sharp}(f) \mathscr{U}_{\mathrm{gen}}(t ; s), a^{\mathrm{b}}(h)\right], \mathscr{M}\right] \psi\right\| .
$$


Here, $a^{\sharp}, a^{b}$ are either creation or annihilation operators. Since $e^{-i \mathscr{K} t} a^{\sharp}(f) e^{i \mathscr{K} t}=$ $a^{\sharp}\left(e^{i t \Delta} f\right)$ and $\left\|e^{i t \Delta} f\right\|_{2}=\|f\|_{2}$ for all $f \in L^{2}\left(\mathbb{R}^{3}\right)$, we can write

$$
F(t)=\sum_{\sharp} \sup _{\|f\|_{2} \leq 1}\left\|\left[\left[\mathscr{U}_{\mathrm{gen}}^{*}(t ; s) e^{-i \mathscr{K}(t-s)} a^{\sharp}(f) e^{i \mathscr{K}(t-s)} \mathscr{U}_{\mathrm{gen}}(t ; s), a^{\mathrm{b}}(h)\right], \mathscr{M}\right] \psi\right\| .
$$

The commutation relations imply that $F(s)=0$. Furthermore,

$$
\begin{aligned}
i \frac{d}{d t} & {\left[\left[\mathscr{U}_{\operatorname{gen}}^{*}(t ; s) e^{-i \mathscr{K}(t-s)} a^{\sharp}(f) e^{i \mathscr{K}(t-s)} \mathscr{U}_{\operatorname{gen}}(t ; s), a^{\mathrm{b}}(h)\right], \mathscr{M}\right] \psi } \\
& =\left[\left[\mathscr{U}_{\operatorname{gen}}^{*}(t ; s)\left[\left(\mathscr{G}_{\operatorname{gen}, t}-\mathscr{K}\right), e^{-i \mathscr{K}(t-s)} a^{\sharp}(f) e^{i \mathscr{K}(t-s)}\right] \mathscr{U}_{\operatorname{gen}}(t ; s), a^{\mathrm{b}}(h)\right], \mathscr{M}\right] \psi \\
& =\left[\left[\mathscr{U}_{\operatorname{gen}}^{*}(t ; s)\left[\left(\mathscr{G}_{\operatorname{gen}, t}-\mathscr{K}\right), a^{\sharp}\left(e^{-i \Delta(t-s)} f\right)\right] \mathscr{U}_{\operatorname{gen}}(t ; s), a^{\mathrm{b}}(h)\right], \mathscr{M}\right] \psi,
\end{aligned}
$$

using the notation $f_{t}=e^{-i \Delta t} f$. Analogous calculations as in the proof of Lemma 3 show that

$$
\left[\left(\mathscr{G}_{\text {gen }, t}-\mathscr{K}\right), a^{\sharp}\left(f_{t}\right)\right]=a\left(h_{1, t}\right)+a^{*}\left(h_{2, t}\right) .
$$

The assumption (2.3) implies $\left\|h_{i, t}\right\|_{2} \leq C e^{C|t|}\|f\|_{2}$ for $i=1,2$. Thus,

$$
\left\|\left[\left[\mathscr{U}_{\text {gen }}^{*}(t ; s) a^{\sharp}(f) \mathscr{U}_{\mathrm{gen}}(t ; s), a^{b}(g)\right], \mathscr{M}\right] \psi\right\| \leq C \int_{0}^{t} d \tau e^{C|\tau|} F(\tau)
$$

for all $f \in L^{2}\left(\mathbb{R}^{3}\right)$ and therefore

$$
0 \leq F(t) \leq C \int_{0}^{t} \mathrm{~d} \tau e^{C|\tau|} F(\tau)
$$

Since $F(s)=0$, the Gronwall inequality implies $F(t)=0$ for all $t \in \mathbb{R}$. Hence,

$$
\left[\left[\mathscr{U}_{\mathrm{gen}}^{*}(t ; s) A\left(f_{1}, h_{1}\right) \mathscr{U}_{\mathrm{gen}}(t ; s), A\left(f_{2}, h_{2}\right)\right], \mathscr{M}\right]=0
$$

for every $f_{1}, f_{2}, h_{1}, h_{2} \in L^{2}\left(\mathbb{R}^{3}\right)$ and every bounded operator $\mathscr{M}$ on the Fock space $\mathscr{F}$ such that $\mathscr{M} \mathscr{D}(\mathscr{K}+\mathscr{N}) \subset \mathscr{D}(\mathscr{K}+\mathscr{N})$. We claim that

$$
\begin{aligned}
\langle\psi & \left.,\left[\mathscr{U}_{\mathrm{gen}}^{*}(t ; s) A\left(f_{1}, h_{1}\right) \mathscr{U}_{\mathrm{gen}}(t ; s), A\left(f_{2}, h_{2}\right)\right] \psi\right\rangle \\
= & \left\langle\Omega,\left[\mathscr{U}_{\mathrm{gen}}^{*}(t ; s) A\left(f_{1}, h_{1}\right) \mathscr{U}_{\mathrm{gen}}(t ; s), A\left(f_{2}, h_{2}\right)\right] \Omega\right\rangle
\end{aligned}
$$

for all $\psi \in \mathscr{D}(\mathscr{K}+\mathscr{N})$ with $\|\psi\|=1$. Combining (2.9) with (2.11), we find

$$
\begin{gathered}
\left\langle\psi,\left[\mathscr{U}_{\text {gen }}(t ; s) A\left(f_{1}, h_{1}\right) \mathscr{U}_{\text {gen }}(t ; s), A\left(f_{2}, h_{2}\right)\right] \psi\right\rangle \\
=\left\langle\Omega,\left[A\left(\Theta(t ; s)\left(f_{1}, h_{1}\right)\right), A\left(f_{2}, h_{2}\right)\right] \Omega\right\rangle
\end{gathered}
$$




$$
=\left(\Theta(t ; s)\left(f_{1}, h_{1}\right), S\left(f_{2}, h_{2}\right)\right)_{L^{2} \oplus L^{2}}
$$

where $S$ is defined in 1.34 . It follows that

$$
\left[\mathscr{U}_{\mathrm{gen}}^{*}(t ; s) A\left(f_{1}, h_{1}\right) \mathscr{U}_{\mathrm{gen}}(t ; s)-A\left(\Theta\left(f_{1}, h_{1}\right)\right), A\left(f_{2}, h_{2}\right)\right]=0,
$$

for all $f_{1}, h_{1}, f_{2}, h_{2} \in L^{2}\left(\mathbb{R}^{3}\right)$. Consider now

$$
R:=\mathscr{U}_{\text {gen }}^{*}(t ; s) A\left(f_{1}, h_{1}\right) \mathscr{U}_{\text {gen }}(t ; s)-A\left(\Theta\left(f_{1}, h_{1}\right)\right) .
$$

On the one hand, (2.9) shows that $R \Omega=0$, and on the other hand, it follows from (2.12), that $R$ commutes with any creation and annihilation operator. Since states of the form $a^{*}\left(f_{1}\right) \ldots a^{*}\left(f_{n}\right) \Omega$ build a basis of the Fock space $\mathscr{F}$, we conclude

$$
\mathscr{U}_{\mathrm{gen}}^{*}(t ; s) A(f, h) \mathscr{U}_{\mathrm{gen}}(t ; s)=A(\Theta(t ; s)(f, h))
$$

for all $f, g \in L^{2}\left(\mathbb{R}^{3}\right)$.

Now, we are left with proving (2.11). For this, note that (2.10) implies

$$
\begin{aligned}
& {\left[\left[\mathscr{U}_{\text {gen }}^{*}(t ; s) A\left(f_{1}, h_{1}\right) \mathscr{U}_{\text {gen }}(t ; s), A\left(f_{2}, h_{2}\right)\right], P_{\psi}\right]} \\
& \quad=\left[\left[\mathscr{U}_{\text {gen }}^{*}(t ; s) A\left(f_{1}, h_{1}\right) \mathscr{U}_{\mathrm{gen}}(t ; s), A\left(f_{2}, h_{2}\right)\right], P_{\Omega}\right]=0,
\end{aligned}
$$

where $P_{\psi}$ resp. $P_{\Omega}$ denote the projection on the subspace of $\mathscr{F}$ spanned by $\psi$ resp. $\Omega$. Therefore, on the one hand

$$
\begin{aligned}
\langle\psi & \left.,\left[\mathscr{U}_{\mathrm{gen}}^{*}(t ; s) A\left(f_{1}, h_{1}\right) \mathscr{U}_{\mathrm{gen}}(t ; s), A\left(f_{2}, h_{2}\right)\right] \Omega\right\rangle \\
& =\left\langle\psi,\left[\mathscr{U}_{\mathrm{gen}}^{*}(t ; s) A\left(f_{1}, h_{1}\right) \mathscr{U}_{\mathrm{gen}}(t ; s), A\left(f_{2}, h_{2}\right)\right] P_{\psi} \Omega\right\rangle \\
& =\left\langle\psi,\left[\mathscr{U}_{\mathrm{gen}}^{*}(t ; s) A\left(f_{1}, h_{1}\right) \mathscr{U}_{\mathrm{gen}}(t ; s), A\left(f_{2}, h_{2}\right)\right] \psi\right\rangle\langle\psi, \Omega\rangle
\end{aligned}
$$

and on the other hand,

$$
\begin{aligned}
\langle\psi & \left.,\left[\mathscr{U}_{\mathrm{gen}}^{*}(t ; s) A\left(f_{1}, h_{1}\right) \mathscr{U}_{\mathrm{gen}}(t ; s), A\left(f_{2}, h_{2}\right)\right] \Omega\right\rangle \\
& =\langle\psi, \Omega\rangle\left\langle\Omega,\left[\mathscr{U}_{\mathrm{gen}}^{*}(t ; s) A\left(f_{1}, h_{1}\right) \mathscr{U}_{\mathrm{gen}}(t ; s), A\left(f_{2}, h_{2}\right)\right] \Omega\right\rangle .
\end{aligned}
$$

Assuming that $\langle\psi, \Omega\rangle \neq 0$, claim (2.11) follows. If $\langle\psi, \Omega\rangle=0$, we repeat the same arguments with $\widetilde{\psi}=\frac{1}{\sqrt{2}}(\psi+\Omega)$. This leads to (2.11).

It remains to prove the properties (1.35). Since for all $f, g \in L^{2}\left(\mathbb{R}^{3}\right)$

$$
\begin{aligned}
(A(\Theta(t ; s)(f, h)))^{*} & =\left(\mathscr{U}_{\mathrm{gen}}^{*}(t ; s) A(f, h) \mathscr{U}_{\mathrm{gen}}(t ; s)\right)^{*} \\
& =\mathscr{U}_{\mathrm{gen}}^{*}(t ; s) A(f, h)^{*} \mathscr{U}_{\mathrm{gen}}(t ; s)
\end{aligned}
$$




$$
\begin{aligned}
& =\mathscr{U}_{\text {gen }}^{*}(t ; s) A(J f, J h) \mathscr{U}_{\text {gen }}(t ; s) \\
& =A(\Theta(t ; s)(J f, J h)),
\end{aligned}
$$

the first property follows. Furthermore, from

$$
\begin{aligned}
{[} & \left.A\left(\Theta(t ; s)\left(f_{1}, h_{1}\right)\right), A\left(\Theta(t ; s)\left(f_{2}, h_{2}\right)\right)\right] \\
& =\left[\mathscr{U}_{\text {gen }}^{*}(t ; s) A\left(f_{1}, h_{2}\right) \mathscr{U}_{\text {gen }}(t ; s), \mathscr{U}^{*}(t ; s) A\left(f_{2}, h_{2}\right) \mathscr{U}(t ; s)\right] \\
& =\mathscr{U}_{\text {gen }}^{*}(t ; s)\left[A\left(f_{1}, h_{1}\right), A\left(f_{2}, h_{2}\right)\right] \mathscr{U}_{\mathrm{gen}}(t ; s) \\
& =\left\langle\left(f_{1}, h_{1}\right), S\left(f_{2}, h_{2}\right)\right\rangle,
\end{aligned}
$$

we deduce the second property.

\subsection{Proof of Theorem 1}

The proof uses ideas introduced in [32]. We consider the expectation value

$$
\begin{aligned}
& \mathbb{E}_{\Psi_{N, t}}\left[g_{1}\left(\mathscr{O}_{1, N, t}\right) \ldots g_{k}\left(\mathscr{O}_{k, N, t}\right)\right] \\
& \quad=\left\langle\Psi_{N, t}, g_{1}\left(\mathscr{O}_{1, N, t}\right) \ldots g_{k}\left(\mathscr{O}_{k, N, t}\right) \Psi_{N, t}\right\rangle \\
& \quad=\int \mathrm{d} s_{1} \ldots \mathrm{d} s_{k} \widehat{g}_{1}\left(s_{1}\right) \ldots \widehat{g}_{k}\left(s_{k}\right)\left\langle\Psi_{N, t}, e^{i s_{1} \mathscr{O}_{1, N, t}} \ldots e^{i s_{k} \mathscr{O}_{k, N, t}} \Psi_{N, t}\right\rangle
\end{aligned}
$$

The norm approximation (1.31) from [11] implies that for every $\alpha<\min \{\beta / 2$, (1$\beta) / 2$ \} there exists $C>0$ such that

$$
\begin{aligned}
& \mid \mathbb{E}_{\Psi_{N, t}}\left[g_{1}\left(\mathscr{O}_{1, N, t}\right) \ldots g_{k}\left(\mathscr{O}_{k, N, t}\right)\right] \\
& \quad-\int \mathrm{d} s_{1} \ldots \mathrm{d} s_{k} \widehat{g}_{1}\left(s_{1}\right) \ldots \widehat{g}_{k}\left(s_{k}\right) \\
& \quad \times\left\langle\mathscr{U}_{\varphi_{N, t}^{*}}^{*} T_{N, t}^{*} \mathscr{U}_{2}(t ; 0) \Omega, e^{i s_{1} \mathscr{O}_{1, N, t}} \ldots e^{i s_{k} \mathscr{O}_{k, N, t}} \mathscr{U}_{\varphi_{N, t}}^{*} T_{N, t}^{*} \mathscr{U}_{2}(t ; 0) \Omega\right\rangle \mid \\
& \leq C N^{-\gamma} \prod_{j=1}^{k}\left\|\widehat{g}_{j}\right\|_{1} .
\end{aligned}
$$

We are hence left with computing the expectation value

$$
\left\langle\mathscr{U}_{\varphi_{N, t}}^{*} T_{N, t}^{*} \mathscr{U}_{2}(t ; 0) \Omega, e^{i s_{1} \mathscr{O}_{1, N, t}} \ldots e^{i s_{k} \mathscr{O}_{k, N, t}} \mathscr{U}_{\varphi_{N, t}}^{*} T_{N, t}^{*} \mathscr{U}_{2}(t ; 0) \Omega\right\rangle .
$$

We split this computation in several Lemmata.

Lemma 4 (Action of the unitary $\left.\mathscr{U}_{\varphi_{N, t}}\right)$ Let $T_{N, t}$ and $\mathscr{U}_{2}(t ; 0)$ be as defined in (1.13) resp. (1.26). Moreover, let $\xi_{N, t}=T_{N, t}^{*} \mathscr{U}_{2}(t ; 0) \Omega$. Then, using the same notations as in Theorem 1, there exists $C>0$ such that 


$$
\begin{aligned}
& \mid\left\langle\mathscr{U}_{\varphi_{N, t}} \xi_{N, t}, e^{i s_{1} \mathscr{O}_{1, N, t}} \ldots e^{\left.i s_{k} \mathscr{O}_{k, N, t} \mathscr{U}_{\varphi_{N, t}} \xi_{N, t}\right\rangle}\right. \\
& \quad-\left\langle\xi_{N, t}, e^{i s_{1} \phi_{b}\left(q_{N, t} O_{1} \varphi_{N, t}\right)} \ldots e^{i s_{k} \phi_{b}\left(q_{N, t} O_{k} \varphi_{N, t}\right)} \xi_{N, t}\right\rangle \mid \\
& \quad \leq \frac{C}{\sqrt{N}} \sum_{m=1}^{k}\left|s_{m}\right|\left\|O_{m}\right\|\left(1+\sum_{j=m}^{k} s_{j}^{2}\left\|O_{j}\right\|^{2}\right)
\end{aligned}
$$

Proof We recall that for $f \in L^{2}\left(\mathbb{R}^{3}\right)$ we denote $\phi_{b}(f)=b^{*}(f)+b(f)$ with the modified creation and annihilation operators $b^{*}(f), b(f)$ defined in (1.9).

In order to show Lemma 4 , we define for $j \in\{1, \ldots, k\}$

$$
\widetilde{O}_{j, N, t}=O_{j}-\left\langle\varphi_{N, t}, O_{j} \varphi_{N, t}\right\rangle
$$

We observe that

$$
\mathscr{O}_{j, N, t}=\frac{1}{\sqrt{N}}\left[\mathrm{~d} \Gamma\left(q_{N, t} \widetilde{O}_{j, N, t} q_{N, t}\right)+\mathrm{d} \Gamma\left(p_{N, t} O_{j} q_{N, t}\right)+\mathrm{d} \Gamma\left(q_{N, t} O_{j} p_{N, t}\right)\right],
$$

where $p_{N, t}=\left|\varphi_{N, t}\right\rangle\left\langle\varphi_{N, t}\right|$ and $q_{N, t}=1-p_{N, t}$. The properties (1.8) of the unitary $\mathscr{U}_{\varphi_{N, t}}$ imply

$$
\mathscr{U}_{\varphi_{N, t}}^{*} \mathscr{O}_{j, N, t} \mathscr{U}_{\varphi_{N, t}}=\frac{1}{\sqrt{N}} \mathrm{~d} \Gamma\left(q_{N, t} \widetilde{O}_{j, N, t} q_{N, t}\right)+\phi_{b}\left(q_{N, t} O_{j} \varphi_{N, t}\right) .
$$

Hence,

$$
\begin{aligned}
& \left\langle\mathscr{U}_{\varphi_{N, t}} \xi_{N, t}, e^{i s_{1} \mathscr{O}_{1, N, t}} \ldots e^{i s_{k} \mathscr{O}_{k, N, t}} \mathscr{U}_{\varphi_{N, t}} \xi_{N, t}\right\rangle \\
& \quad=\left\langle\xi_{N, t}, \prod_{j=1}^{k} e^{\left.i s_{j}\left(\frac{1}{\sqrt{N}} \mathrm{~d} \Gamma\left(q_{N, t} \widetilde{O}_{j, N, t} q_{N, t}\right)+\phi_{b}\left(q_{N, t} O_{j} \varphi_{N, t}\right)\right)_{\xi_{N, t}}\right\rangle .}\right.
\end{aligned}
$$

We compute

$$
\begin{aligned}
& \left\langle\mathscr{U}_{\varphi_{N, t}} \xi_{N, t}, e^{i s_{1} \mathscr{O}_{1, N, t}} \ldots e^{i s_{k} \mathscr{O}_{k, N, t}} \mathscr{U}_{\varphi_{N, t}} \xi_{N, t}\right\rangle \\
& \quad-\left\langle\xi_{N, t}, e^{i s_{1} \phi_{b}\left(q_{N, t} O_{1} \varphi_{N, t}\right)} \ldots e^{i s_{k} \phi_{b}\left(q_{N, t} O_{k} \varphi_{N, t}\right)} \xi_{N, t}\right\rangle \\
& =\sum_{m=1}^{k}\left\langle\xi_{N, t}, \prod_{j=1}^{m-1} e^{i s_{j}\left(\frac{1}{\sqrt{N}} \mathrm{~d} \Gamma\left(q_{N, t} \widetilde{O}_{j, N, t} q_{N, t}\right)+\phi_{b}\left(q_{N, t} O_{j} \varphi_{N, t}\right)\right)}\right. \\
& \quad \times\left(e^{i s_{m}\left(\frac{1}{\sqrt{N}} \mathrm{~d} \Gamma\left(q_{N, t} \widetilde{O}_{m, N, t} q_{N, t}\right)+\phi_{b}\left(q_{N, t} O_{m} \varphi_{N, t}\right)\right)}-e^{i s_{m} \phi_{b}\left(q_{N, t} O_{m} \varphi_{N, t}\right)}\right) \\
& \left.\quad \times \prod_{j=m+1}^{k} e^{i s_{j} \phi_{b}\left(q_{N, t} O_{j} \varphi_{N, t}\right)} \xi_{N, t}\right\rangle .
\end{aligned}
$$


Using the fundamental theorem of calculus, we can write the difference as an integral

$$
\begin{aligned}
& \left\langle\mathscr{U}_{\varphi_{N, t}} \xi_{N, t}, e^{i s_{1} \mathscr{O}_{1, N, t}} \ldots e^{i s_{k} \mathscr{O}_{k, N, t}} \mathscr{U}_{\varphi_{N, t}} \xi_{N, t}\right\rangle \\
& \quad-\left\langle\xi_{N, t}, e^{i s_{1} \phi_{b}\left(q_{N, t} O_{1} \varphi_{N, t}\right)} \ldots e^{i s_{k} \phi_{b}\left(q_{N, t} O_{k} \varphi_{N, t}\right)} \xi_{N, t}\right\rangle \\
& =\frac{1}{\sqrt{N}} \sum_{m=1}^{k} \int_{0}^{s_{m}} \mathrm{~d} \tau\left\langle\xi_{N, t}, \prod_{j=1}^{m-1} e^{i s_{j}\left(\frac{1}{\sqrt{N}} \mathrm{~d} \Gamma\left(q_{N, t} \widetilde{O}_{j, N, t} q_{N, t}\right)+\phi_{b}\left(q_{N, t} O_{j} \varphi_{N, t}\right)\right)}\right. \\
& \quad \times e^{i \tau\left(\frac{1}{\sqrt{N}} \mathrm{~d} \Gamma\left(q_{N, t} \widetilde{O}_{m, N, t} q_{N, t}\right)+\phi_{b}\left(q_{N, t} O_{m} \varphi_{N, t}\right)\right)} \mathrm{d} \Gamma\left(q_{N, t} \widetilde{O}_{m, N, t} q_{N, t}\right) \\
& \left.\quad \times e^{i(1-\tau) \phi_{b}\left(q_{N, t} O_{m} \varphi_{N, t}\right)} \prod_{j=m+1}^{k} e^{i s_{j} \phi_{b}\left(q_{N, t} O_{j} \varphi_{N, t}\right)} \xi_{N, t}\right\rangle .
\end{aligned}
$$

The estimate $\|\mathrm{d} \Gamma(A) \psi\| \leq\|A\|\|\mathscr{N} \psi\|$ leads to

$$
\begin{aligned}
& \mid\left\langle\mathscr{U}_{\varphi_{N, t}} \xi_{N, t}, e^{i s_{1} \mathscr{O}_{1, N, t}} \ldots e^{i s_{k} \mathscr{O}_{k, N, t}} \mathscr{U}_{\varphi_{N, t}} \xi_{N, t}\right\rangle \\
& \quad-\left\langle\xi_{N, t}, e^{i s_{1} \phi_{b}\left(q_{N, t} O_{1} \varphi_{N, t}\right)} \ldots e^{i s_{k} \phi_{b}\left(q_{N, t} O_{k} \varphi_{N, t}\right)} \xi_{N, t}\right\rangle \mid \\
& \leq \frac{1}{\sqrt{N}} \sum_{m=1}^{k} \int_{0}^{s_{m}} \mathrm{~d} \tau\left\|q_{N, t} \widetilde{O}_{m, N, t} q_{N, t}\right\| \\
& \quad \times\left\|\mathscr{N} e^{i(1-\tau) \phi_{b}\left(q_{N, t} O_{m} \varphi_{N, t}\right)} \prod_{j=m+1}^{k} e^{i s_{j} \phi_{b}\left(q_{N, t} O_{j} \varphi_{N, t}\right)} \xi_{N, t}\right\| .
\end{aligned}
$$

Since $\left\|q_{N, t} \widetilde{O}_{m, N, t} q_{N, t}\right\| \leq\left\|O_{m}\right\|$ and $\left\|q_{N, t} O_{j} \varphi_{N, t}\right\| \leq\left\|O_{j}\right\|$, we find with (2.8)

$$
\begin{aligned}
& \mid\left\langle\mathscr{U}_{\varphi_{N, t}} \xi_{N, t}, e^{i s_{1} \mathscr{O}_{1, N, t}} \ldots e^{i s_{k} \mathscr{O}_{k, N, t}} \mathscr{U}_{\varphi_{N, t}} \xi_{N, t}\right\rangle \\
& \quad-\left\langle\xi_{N, t}, e^{i s_{1} \phi_{b}\left(q_{N, t} O_{1} \varphi_{N, t}\right)} \ldots e^{i s_{k} \phi_{b}\left(q_{N, t} O_{k} \varphi_{N, t}\right)} \xi_{N, t}\right\rangle \mid \\
& \quad \leq \frac{1}{\sqrt{N}} \sum_{m=1}^{k}\left\|O_{m}\right\| \int_{0}^{s_{m}} \mathrm{~d} \tau\left\|\left(\mathscr{N}_{+}+(1-\tau)^{2}\left\|O_{m}\right\|^{2}+\sum_{j=m+1}^{k} s_{j}^{2}\left\|O_{j}\right\|^{2}+\alpha\right) \xi_{N, t}\right\|
\end{aligned}
$$

for $\alpha \geq 1$. Recall that $\xi_{N, t}=T_{N, t}^{*} \mathscr{U}_{2}(t ; 0) \Omega$. It follows from (2.5) and Lemma 2 that

$$
\left\langle\xi_{N, t}, \mathscr{N}_{+}^{2} \xi_{N, t}\right\rangle \leq C
$$


for a constant $C>0$ uniform in $N$. Hence,

$$
\begin{aligned}
& \mid\left\langle\mathscr{U}_{\varphi_{N, t}} \xi_{N, t}, e^{i s_{1} \mathscr{O}_{1, N, t}} \ldots e^{i s_{k} \mathscr{O}_{k, N, t}} \mathscr{U}_{\varphi_{N, t}} \xi_{N, t}\right\rangle \\
& \quad-\left\langle\xi_{N, t}, e^{i s_{1} \phi_{b}\left(q_{N, t} O_{1} \varphi_{N, t}\right)} \ldots e^{i s_{k} \phi_{b}\left(q_{N, t} O_{k} \varphi_{N, t}\right)} \xi_{N, t}\right\rangle \mid \\
& \quad \leq \frac{C}{\sqrt{N}} \sum_{m=1}^{k}\left|s_{m}\right|\left\|O_{m}\right\|\left(1+\sum_{j=m}^{k} s_{j}^{2}\left\|O_{j}\right\|^{2}\right)
\end{aligned}
$$

Lemma 5 ( Replace modified creation and annihilation operators with standard ones) Let $T_{N, t}$ and $\mathscr{U}_{2}(t ; 0)$ be as defined in (1.13) resp. (1.26). Moreover, let $\xi_{N, t}=$ $T_{N, t}^{*} \mathscr{U}_{2}(t ; 0) \Omega$. Then, with the same notations as in Theorem 1 , there exists $C>0$ such that

$$
\begin{aligned}
& \mid\left\langle\xi_{N, t}, e^{i s_{1} \phi_{b}\left(q_{N, t} O_{1} \varphi_{N, t}\right)} \ldots e^{i s_{k} \phi_{b}\left(q_{N, t} O_{k} \varphi_{N, t}\right)} \xi_{N, t}\right\rangle \\
& \quad-\left\langle\xi_{N, t}, e^{i s_{1} \phi_{a}\left(q_{N, t} O_{1} \varphi_{N, t}\right)} \ldots e^{i s_{k} \phi_{a}\left(q_{N, t} O_{k} \varphi_{N, t}\right)} \xi_{N, t}\right\rangle \mid \\
& \quad \leq \frac{C}{N} \sum_{m=1}^{k}\left\|O_{m}\right\|\left|s_{m}\right|\left(1+\sum_{j=m}^{k} s_{j}^{2}\left\|O_{j}\right\|\right)^{3 / 2} .
\end{aligned}
$$

Proof Recall that

$$
\phi_{a}(f)=a^{*}(f)+a(f)
$$

with the standard creation and annihilation operators $a^{*}(f), a(f)$, while

$$
\phi_{b}(f)=b^{*}(f)+b(f)
$$

with the modified creation and annihilation operators defined in (1.9). To this end, we compute

$$
\begin{aligned}
\left\langle\xi_{N, t},\right. & \left.e^{i s_{1} \phi_{b}\left(q_{N, t} O_{1} \varphi_{N, t}\right)} \ldots e^{i s_{k} \phi_{b}\left(q_{N, t} O_{k} \varphi_{N, t}\right)} \xi_{N, t}\right\rangle \\
& -\left\langle\xi_{N, t}, e^{i s_{1} \phi_{a}\left(q_{N, t} O_{1} \varphi_{N, t}\right)} \ldots e^{i s_{k} \phi_{a}\left(q_{N, t} O_{k} \varphi_{N, t}\right)} \xi_{N, t}^{(1)}\right\rangle \\
= & \sum_{m=1}^{k}\left\langle\xi_{N, t}, \prod_{j=1}^{m-1} e^{i s_{j} \phi_{b}\left(q_{N, t} O_{j} \varphi_{N, t}\right)}\right. \\
& \times\left(e^{i s_{m} \phi_{b}\left(q_{N, t} O_{m} \varphi_{N, t}\right)}-e^{i s_{m} \phi_{a}\left(q_{N, t} O_{m} \varphi_{N, t}\right)}\right)
\end{aligned}
$$




$$
\begin{aligned}
& \left.\times \prod_{j=m+1}^{k} e^{i s_{j} \phi_{a}\left(q_{N, t} O_{j} \varphi_{N, t}\right)} \xi_{N, t}\right\rangle \\
= & \sum_{m=1}^{k} \int_{0}^{s_{m}} \mathrm{~d} \tau\left\langle\xi_{N, t}, \prod_{j=1}^{m-1} e^{i s_{j} \phi_{b}\left(q_{N, t} O_{j} \varphi_{N, t}\right)} e^{i \tau \phi_{b}\left(q_{N, t} O_{m} \varphi_{N, t}\right)}\right. \\
& \times\left(\phi_{b}\left(q_{N, t} O_{m} \varphi_{N, t}\right)-\phi_{a}\left(q_{N, t} O_{m} \varphi_{N, t}\right)\right) \\
& \left.\times e^{i(1-\tau) \phi_{a}\left(q_{N, t} O_{m} \varphi_{N, t}\right)} \prod_{j=m+1}^{k} e^{i s_{j} \phi_{a}\left(q_{N, t} O_{j} \varphi_{N, t}\right)} \xi_{N, t}\right\rangle .
\end{aligned}
$$

By definition of the modified creation and annihilation operators (1.9), we obtain

$$
\begin{aligned}
& \left\langle\xi_{N, t}, e^{i s_{1} \phi_{b}\left(q_{N, t} O_{1} \varphi_{N, t}\right)} \ldots e^{i s_{k} \phi_{b}\left(q_{N, t} O_{k} \varphi_{N, t}\right)} \xi_{N, t}\right\rangle \\
& -\left\langle\xi_{N, t}, e^{i s_{1} \phi_{a}\left(q_{N, t} O_{1} \varphi_{N, t}\right)} \ldots e^{i s_{k} \phi_{a}\left(q_{N, t} O_{k} \varphi_{N, t}\right)} \xi_{N, t}\right\rangle \\
& =\sum_{m=1}^{k} \int_{0}^{s_{m}} \mathrm{~d} \tau\left\langle\xi_{N, t}^{(1)}, \prod_{j=1}^{m-1} e^{i s_{j} \phi_{b}\left(q_{N, t} O_{j} \varphi_{N, t}\right)} e^{i \tau \phi_{b}\left(q_{N, t} O_{m} \varphi_{N, t}\right)}\right. \\
& \times a^{*}\left(q_{N, t} O_{m} \varphi_{N, t}\right)\left(\sqrt{1-\mathscr{N}_{+} / N}-1\right) \\
& \left.\times e^{i(1-\tau) \phi_{a}\left(q_{N, t} O_{m} \varphi_{N, t}\right)} \prod_{j=m+1}^{k} e^{i s_{j} \phi_{a}\left(q_{N, t} O_{j} \varphi_{N, t}\right)} \xi_{N, t}\right\rangle \\
& +\sum_{m=1}^{k} \int_{0}^{s_{m}} \mathrm{~d} \tau\left\langle\xi_{N, t}, \prod_{j=1}^{m-1} e^{i s_{j} \phi_{b}\left(q_{N, t} O_{j} \varphi_{N, t}\right)} e^{i \tau \phi_{b}\left(q_{N, t} O_{m} \varphi_{N, t}\right)}\right. \\
& \times\left(\sqrt{1-\mathscr{N}_{+} / N}-1\right) a\left(q_{N, t} O_{m} \varphi_{N, t}\right) \\
& \left.\times e^{i(1-\tau) \phi_{a}\left(q_{N, t} O_{m} \varphi_{N, t}\right)} \prod_{j=m+1}^{k} e^{i s_{j} \phi_{a}\left(q_{N, t} O_{j} \varphi_{N, t}\right)} \xi_{N, t}\right\rangle .
\end{aligned}
$$

Since $\left\|a^{*}(f) \xi\right\| \leq\|f\|_{2}\left\|(\mathscr{N}+1)^{1 / 2} \xi\right\|$ resp. $\|a(f) \xi\| \leq\|f\|_{2}\left\|\mathscr{N}^{1 / 2} \xi\right\|$ and $\left\|q_{N, t} O_{m} \varphi_{N, t}\right\|_{2} \leq\left\|O_{m}\right\|$, we find

$$
\begin{aligned}
& \mid\left\langle\xi_{N, t}, e^{i s_{1} \phi_{b}\left(q_{N, t} O_{1} \varphi_{N, t}\right)} \ldots e^{i s_{k} \phi_{b}\left(q_{N, t} O_{k} \varphi_{N, t}\right)} \xi_{N, t}\right\rangle \\
& \quad-\left\langle\xi_{N, t}, e^{i s_{1} \phi_{a}\left(q_{N, t} O_{1} \varphi_{N, t}\right)} \ldots e^{i s_{k} \phi_{a}\left(q_{N, t} O_{k} \varphi_{N, t}\right)} \xi_{N, t}\right\rangle \mid \\
& \quad \leq \frac{2}{N} \sum_{m=1}^{k}\left\|O_{m}\right\| \int_{0}^{s_{m}} \mathrm{~d} \tau\left\|(\mathscr{N}+1)^{3 / 2} e^{i(1-\tau) \phi_{a}\left(q_{N, t} O_{m} \varphi_{N, t}\right)} \prod_{j=m+1}^{k} e^{i s_{j} \phi_{a}\left(q_{N, t} O_{j} \varphi_{N, t}\right)} \xi_{N, t}\right\| .
\end{aligned}
$$


Now, Lemma 2 together with (2.7) and (2.5) implies

$$
\begin{aligned}
& \mid\left\langle\xi_{N, t}, e^{i s_{1} \phi_{b}\left(q_{N, t} O_{1} \varphi_{N, t}\right)} \ldots e^{i s_{k} \phi_{b}\left(q_{N, t} O_{k} \varphi_{N, t}\right)} \xi_{N, t}\right\rangle \\
& \quad-\left\langle\xi_{N, t}, e^{i s_{1} \phi_{a}\left(q_{N, t} O_{1} \varphi_{N, t}\right)} \ldots e^{i s_{k} \phi_{a}\left(q_{N, t} O_{k} \varphi_{N, t}\right)} \xi_{N, t}\right\rangle \mid \\
& \quad \leq \frac{C}{N} \sum_{m=1}^{k}\left\|O_{m}\right\|\left|s_{m}\right|\left(1+\sum_{j=m}^{k} s_{j}^{2}\left\|O_{j}\right\|\right)^{3 / 2} .
\end{aligned}
$$

Lemma 6 (Replace modified Hartree equation with nonlinear Schrödinger equation) Let $T_{N, t}$ and $\mathscr{U}_{2}(t ; 0)$ be as defined in (1.13) resp. (1.26). Moreover, let $\xi_{N, t}=$ $T_{N, t}^{*} \mathscr{U}_{2}(t ; 0) \Omega$. Then, with the same notations as in Theorem 1 , there exists $C>0$ such that

$$
\begin{aligned}
& \mid\left\langle\xi_{N, t}, e^{i s_{1} \phi_{a}\left(q_{N, t} O_{1} \varphi_{N, t}\right)} \ldots e^{i s_{k} \phi_{a}\left(q_{N, t} O_{k} \varphi_{N, t}\right)} \xi_{N, t}\right\rangle \\
& \quad-\left\langle\xi_{N, t}^{(1)}, e^{i s_{1} \phi_{a}\left(q_{t} O_{1} \varphi_{t}\right)} \ldots e^{i s_{k} \phi_{a}\left(q_{t} O_{k} \varphi_{t}\right)} \xi_{N, t}^{(1)}\right\rangle \mid \\
& \quad \leq C N^{-\gamma} \sum_{m=1}^{k}\left|s_{m}\right|\left\|O_{m}\right\|\left(1+\sum_{j=m}^{k} s_{j}^{2}\left\|O_{j}\right\|^{2}\right)^{1 / 2} \exp (\exp (C|t|)) .
\end{aligned}
$$

Proof By linearity of the operator $\phi_{a}(f)$, we compute

$$
\begin{aligned}
\left\langle\xi_{N, t},\right. & \left.e^{i s_{1} \phi_{a}\left(q_{N, t} O_{1} \varphi_{N, t}\right)} \ldots e^{i s_{k} \phi_{a}\left(q_{N, t} O_{k} \varphi_{N, t}\right)} \xi_{N, t}\right\rangle \\
& -\left\langle\xi_{N, t}, e^{i s_{1} \phi_{a}\left(q_{t} O_{1} \varphi_{t}\right)} \ldots e^{i s_{k} \phi_{a}\left(q_{t} O_{k} \varphi_{t}\right)} \xi_{N, t}\right\rangle \\
= & \sum_{m=1}^{k}\left\langle\xi_{N, t}, \prod_{j=1}^{m-1} e^{i s_{j} \phi_{a}\left(q_{N, t} O_{j} \varphi_{N, t}\right)}\left(e^{i s_{m} \phi_{a}\left(q_{N, t} O_{m} \varphi_{N, t}\right)}-e^{i s_{m} \phi_{a}\left(q_{t} O_{m} \varphi_{t}\right)}\right)\right. \\
& \left.\times \prod_{j=m+1}^{k} e^{i s_{j} \phi_{a}\left(q_{N, t} O_{j} \varphi_{N, t}\right)} \xi_{N, t}\right\rangle \\
= & \sum_{m=1}^{k} \int_{0}^{s_{m}} \mathrm{~d} \tau\left\langle\xi_{N, t}, \prod_{j=1}^{m-1} e^{i s_{j} \phi_{a}\left(q_{N, t} O_{j} \varphi_{N, t}\right)}\right. \\
& \times e^{i \tau \phi_{a}\left(q_{N, t} O_{m} \varphi_{N, t}\right)} \phi_{a}\left(q_{N, t} O_{m} \varphi_{N, t}-q_{t} O_{m} \varphi_{t}\right) e^{i(1-\tau) \phi_{a}\left(q_{t} O_{m} \varphi_{t}\right)} \\
& \left.\times \prod_{j=m+1}^{k} e^{i s_{j} \phi_{a}\left(q_{N, t} O_{j} \varphi_{N, t}\right)} \xi_{N, t}\right\rangle .
\end{aligned}
$$


As

$$
\begin{aligned}
\left\|q_{N, t} O_{m} \varphi_{N, t}-q_{t} O_{m} \varphi_{t}\right\|_{2} & \leq\left\|O_{m}\right\|\left(\left\|q_{N, t}-q_{t}\right\|_{2}+\left\|\varphi_{N, t}-\varphi_{t}\right\|_{2}\right) \\
& \leq 2\left\|O_{m}\right\|\left\|\varphi_{N, t}-\varphi_{t}\right\|,
\end{aligned}
$$

the estimate (1.18) implies

$$
\left\|q_{N, t} O_{m} \varphi_{N, t}-q_{t} O_{m} \varphi_{t}\right\|_{2} \leq C\left\|O_{m}\right\| N^{-\gamma} \exp (\exp (C|t|))
$$

with $\gamma=\min \{\beta, 1-\beta\}$. Hence, the bound

$$
\left\|\phi_{a}(f) \psi\right\| \leq 2\|f\|_{2}\left\|(\mathscr{N}+1)^{1 / 2} \psi\right\|
$$

leads to

$$
\begin{aligned}
& \mid\left\langle\xi_{N, t}, e^{i s_{1} \phi_{a}\left(q_{N, t} O_{1} \varphi_{N, t}\right)} \ldots e^{i s_{k} \phi_{a}\left(q_{N, t} O_{k} \varphi_{N, t}\right)} \xi_{N, t}\right\rangle \\
& \quad-\left\langle\xi_{N, t}, e^{i s_{1} \phi_{a}\left(q_{t} O_{1} \varphi_{t}\right)} \ldots e^{i s_{k} \phi_{a}\left(q_{t} O_{k} \varphi_{t}\right)} \xi_{N, t}\right\rangle \mid \\
& \leq C N^{-\gamma} \exp (\exp (C|t|)) \sum_{m=1}^{k}\left\|O_{m}\right\| \int_{0}^{s_{m}} \mathrm{~d} \tau \\
& \quad \times\left\|(\mathscr{N}+1)^{1 / 2} e^{i(1-\tau) \phi_{a}\left(q_{t} O_{m} \varphi_{t}\right)} \prod_{j=m+1}^{k} e^{i s_{j} \phi_{a}\left(q_{N, t} O_{j} \varphi_{N, t}\right)} \xi_{N, t}\right\| .
\end{aligned}
$$

We conclude again with Lemma (2.7), Lemma (2.5), and Lemma 2

$$
\begin{aligned}
& \mid\left\langle\xi_{N, t}, e^{i s_{1} \phi_{a}\left(q_{N, t} O_{1} \varphi_{N, t}\right)} \ldots e^{i s_{k} \phi_{a}\left(q_{N, t} O_{k} \varphi_{N, t}\right)} \xi_{N, t}\right\rangle \\
& \quad-\left\langle\xi_{N, t}^{(1)}, e^{i s_{1} \phi_{a}\left(q_{t} O_{1} \varphi_{t}\right)} \ldots e^{i s_{k} \phi_{a}\left(q_{t} O_{k} \varphi_{t}\right)} \xi_{N, t}^{(1)}\right\rangle \mid \\
& \quad \leq C N^{-\gamma} \sum_{m=1}^{k}\left|s_{m}\right|\left\|O_{m}\right\|\left(1+\sum_{j=m}^{k} s_{j}^{2}\left\|O_{j}\right\|^{2}\right)^{1 / 2} \exp (\exp (C|t|)) .
\end{aligned}
$$

Lemma 7 (Action of $T_{N, t}$ ) Let $T_{N, t}$ and $\mathscr{U}_{2}(t ; 0)$ be as defined in (1.13) resp. (1.26). Moreover, let $\xi_{N, t}=T_{N, t}^{*} \mathscr{U}_{2}(t ; 0) \Omega$ and $\xi_{t}=T_{N, t} \xi_{N, t}=\mathscr{U}_{2, t} \Omega$. Then, using the same notations as in Theorem 1, there exists $C>0$ such that 


$$
\begin{aligned}
& \left|\left\langle\xi_{N, t}, e^{i S_{1} \phi_{a}\left(q_{t} O_{1} \varphi_{t}\right)} \ldots e^{i s_{k} \phi_{a}\left(q_{t} O_{k} \varphi_{t}\right)} \xi_{N, t}\right\rangle-\left\langle\xi_{t}, e^{i s_{1} \phi_{a}\left(h_{1, t}\right)} \ldots e^{i s_{k} \phi_{a}\left(h_{k, t}\right)} \xi_{t}\right\rangle\right| \\
& \leq C N^{-\gamma} \sum_{m=1}^{k}\left|s_{m}\right|\left\|O_{m}\right\|\left(1+\sum_{j=m}^{k} s_{j}^{2}\left\|O_{j}\right\|^{2}\right)^{1 / 2} \exp (\exp (C \mid t)) .
\end{aligned}
$$

with $h_{j, t}=\cosh \left(\eta_{t}\right) q_{t} O \varphi_{t}+\sinh \left(\eta_{t}\right) \overline{q_{t} O_{j} \varphi_{t}}$ and $\eta_{t}$ as defined in (1.20).

Proof We compute using the properties (1.14) of the Bogoliubov transformation

$$
T_{N, t}^{*} \phi_{a}\left(q_{t} O_{j} \varphi_{t}\right) T_{N, t}=\phi_{a}\left(\cosh \left(\eta_{N, t}\right) q_{t} O_{j} \varphi_{t}+\sinh \left(\eta_{N, t}\right) \overline{q_{t} O_{j} \varphi_{t}}\right),
$$

with $\eta_{N, t}$ as defined in (1.20). In the following, we denote $h_{j, N, t}=\cosh \left(\eta_{N, t}\right) q_{t} O \varphi_{t}+$ $\sinh \left(\eta_{N, t}\right) \overline{q_{t} O_{j} \varphi_{t}}$. Since

$$
\left\langle\xi_{N, t}, e^{i s_{1} \phi_{a}\left(q_{t} O_{1} \varphi_{t}\right)} \ldots e^{i s_{k} \phi_{a}\left(q_{t} O_{k} \varphi_{t}\right)} \xi_{N, t}\right\rangle=\left\langle\xi_{t}, e^{i s_{1} \phi_{a}\left(h_{1, N, t}\right)} \ldots e^{i s_{k} \phi_{a}\left(h_{k, N, t}\right)} \xi_{t}\right\rangle,
$$

we need to consider

$$
\left\langle\xi_{t}, e^{i s_{1} \phi_{a}\left(h_{1, N, t}\right)} \ldots e^{i s_{k} \phi_{a}\left(h_{k, N, t}\right)} \xi_{t}\right\rangle-\left\langle\xi_{t}, e^{i s_{1} \phi_{a}\left(h_{1, t}\right)} \ldots e^{i s_{k} \phi_{a}\left(h_{k, t}\right)} \xi_{t}\right\rangle
$$

We observe using (1.24)

$$
\begin{aligned}
\left\|h_{j, N, t}-h_{j, t}\right\|_{2} \leq & \left\|O_{m}\right\|\left(\left\|\cosh \left(\eta_{t}\right)-\cosh \left(\eta_{N, t}\right)\right\|_{2}+\| \sinh \left(\eta_{t}\right)-\sinh \left(\eta_{N, t} \|_{2}\right)\right. \\
\leq & 2\left\|O_{m}\right\| \cosh \left(\left(\eta_{N, t}+\eta_{t}\right) / 2\right) \sinh \left(\left(\eta_{N, t}-\eta_{t}\right) / 2\right) \|_{2} \\
& +2\left\|O_{m}\right\| \sinh \left(\left(\eta_{N, t}+\eta_{t}\right) / 2\right) \sinh \left(\left(\eta_{N, t}-\eta_{t}\right) / 2\right) \|_{2} \\
\leq & C\left\|O_{m}\right\|\left\|\eta_{N, t}-\eta_{t}\right\|_{2} .
\end{aligned}
$$

Thus, the estimate (1.22) leads to

$$
\left\|h_{j, N, t}-h_{j, t}\right\|_{2} \leq C N^{-\gamma} \exp (\exp (C|t|)) .
$$

Using $\left\|h_{j, t}\right\|_{2} \leq C\left\|O_{j}\right\|$, the same arguments as in step 3 lead to

$$
\begin{aligned}
& \left|\left\langle\xi_{N, t}, e^{i s_{1} \phi_{a}\left(q_{t} O_{1} \varphi_{t}\right)} \ldots e^{i s_{k} \phi_{a}\left(q_{t} O_{k} \varphi_{t}\right)} \xi_{N, t}\right\rangle-\left\langle\xi_{t}, e^{i s_{1} \phi_{a}\left(h_{1, t}\right)} \ldots e^{i s_{k} \phi_{a}\left(h_{k, t}\right)} \xi_{t}\right\rangle\right| \\
& \quad \leq C N^{-\gamma} \sum_{m=1}^{k}\left|s_{m}\right|\left\|O_{m}\right\|\left(1+\sum_{j=m}^{k} s_{j}^{2}\left\|O_{j}\right\|^{2}\right)^{1 / 2} \exp (\exp (C \mid t)) .
\end{aligned}
$$


Lemma 8 (Computing the expectation value) Let $\mathscr{U}_{2}(t ; 0)$ be as defined in (1.26). Let $\xi_{t}=\mathscr{U}_{2, t} \Omega$. Then, using the same notations as in Theorem 1 ,

$$
\begin{aligned}
& \left\langle\xi_{t}, e^{i s_{1} \phi_{a}\left(h_{1, t}\right)} \ldots e^{i s_{k} \phi_{a}\left(h_{k, t}\right)} \xi_{t}\right\rangle \\
& \quad=\frac{1}{\sqrt{(2 \pi)^{k} \operatorname{det} \Sigma_{t}}} \int d x_{1} \ldots d x_{k} g_{1}\left(x_{1}\right) \ldots g_{k}\left(x_{k}\right) e^{-\frac{1}{2} \sum_{i, j=1}^{k}\left(\Sigma_{t}\right)_{i, j}^{-1} x_{i} x_{j}} .
\end{aligned}
$$

Proof Since $\xi_{t}=\mathscr{U}_{2, t} \Omega$, we are left with computing

$$
\left\langle\xi_{t}, e^{i s_{1} \phi_{a}\left(h_{1, t}\right)} \ldots e^{i s_{k} \phi_{a}\left(h_{k, t}\right)} \xi_{t}\right\rangle=\left\langle\Omega, \mathscr{U}_{2, t}^{*} e^{i s_{1} \phi_{a}\left(h_{1, t}\right)} \ldots e^{i s_{k} \phi_{a}\left(h_{k, t}\right)} \mathscr{U}_{2, t} \Omega\right\rangle
$$

As proven in Proposition 1 , the unitary $\mathscr{U}_{2, t}$ gives rise to a Bogoliubov transformation. Hence, there exists bounded operators $U(t ; 0), V(t ; 0)$ on $L^{2}\left(\mathbb{R}^{3}\right)$ such that

$$
\mathscr{U}_{2, t}^{*} \phi_{a}\left(h_{j, t}\right) \mathscr{U}_{2, t}=\phi_{a}\left(U(t ; 0) h_{j, t}+\overline{V(t ; 0) h}_{j, t}\right) .
$$

In the following, we denote

$$
\begin{aligned}
v_{j, t}= & U(t ; 0) h_{j, t}+{\overline{V(t ; 0) h_{j, t}}}_{=}\left(U(t ; 0) \cosh \eta_{t}+\overline{V(t ; 0)} \sinh \eta_{t}\right) q_{t} O_{j} \varphi_{t} \\
& +\left(U(t ; 0) \sinh \eta_{t}+\overline{V(t ; 0)} \cosh \eta_{t}\right) \overline{q_{t} O_{j} \varphi_{t}}
\end{aligned}
$$

Note that the Baker-Campbell-Hausdorff formula implies on the one hand

$$
e^{i \phi_{a}(f)} e^{i \phi_{a}(g)}=e^{i \phi_{a}(f+g)} e^{-i \operatorname{Im}\langle f, g\rangle}
$$

for $f, g \in L^{2}\left(\mathbb{R}^{3}\right)$, i.e.,

$$
\prod_{j=1}^{k} e^{i s_{j} \phi_{a}\left(v_{j, t}\right)}=e^{i \phi_{a}\left(v_{t}\right)} \prod_{i<j}^{k} e^{-i s_{i} s_{j} \operatorname{Im}\left\langle v_{i, t}, v_{j, t}\right\rangle}
$$

with $v_{t}=\sum_{j=1}^{k} v_{j, t}$. On the other hand, the Baker-Campbell-Hausdorff formula applied to the creation and annihilation operator implies

$$
\prod_{j=1}^{k} e^{i s_{j} \phi_{a}\left(v_{j, t}\right)}=e^{-\left\|v_{t}\right\|_{2}^{2} / 2} e^{a^{*}\left(v_{t}\right)} e^{a\left(v_{t}\right)} \prod_{i<j}^{k} e^{-i s_{i} s_{j} \operatorname{Im}\left\langle v_{i, t}, v_{j, t}\right\rangle}
$$


Hence, we write the expectation value (2.14) as

$$
\begin{aligned}
\left\langle\xi_{t}, e^{i \phi_{a}\left(h_{1, t}\right)} \ldots e^{i s_{k} \phi_{a}\left(h_{k, t}\right)} \xi_{t}\right\rangle & =e^{-\left\|v_{t}\right\|_{2}^{2}} \prod_{i<j}^{k} e^{-i s_{i} s_{j} \operatorname{Im}\left\langle v_{i, t}, v_{j, t}\right\rangle}\left\langle\Omega, e^{a^{*}\left(v_{t}\right)} e^{a\left(v_{t}\right)} \Omega\right\rangle \\
& =e^{-\left\|v_{t}\right\|_{2}^{2}} \prod_{i<j}^{k} e^{-i s_{i} s_{j} \operatorname{Im}\left\langle v_{i, t}, v_{j, t}\right\rangle} .
\end{aligned}
$$

Let $\Sigma_{t} \in \mathbb{C}^{k \times k}$ be given through

$$
\left(\Sigma_{t}\right)_{i, j}= \begin{cases}\left\langle v_{i, t}, v_{j, t}\right\rangle, & \text { if } i<j \\ \left\langle v_{j, t}, v_{i, t}\right\rangle, & \text { otherwise }\end{cases}
$$

then

$$
\left\langle\xi_{t}, e^{i s_{1} \phi_{a}\left(h_{1, t}\right)} \ldots e^{i s_{k} \phi_{a}\left(h_{k, t}\right)} \xi_{t}\right\rangle=e^{-\frac{1}{2} \sum_{i, j=1}^{k}\left(\Sigma_{t}\right)_{i, j} s_{i} s_{j}}
$$

By assumption, the matrix $\Sigma_{t}$ is invertible. Hence,

$$
\begin{aligned}
& \int \mathrm{d} s_{1} \ldots \mathrm{d} s_{k} \widehat{g}_{1}\left(s_{1}\right) \ldots \widehat{g}_{k}\left(s_{k}\right)\left\langle\xi_{t}, e^{i s_{1} \phi_{a}\left(h_{1, t}\right)} \ldots e^{i s_{k} \phi_{a}\left(h_{k, t}\right)} \xi_{t}\right\rangle \\
& =\int \mathrm{d} s_{1} \ldots \mathrm{d} s_{k} \widehat{g}_{1}\left(s_{1}\right) \ldots \widehat{g}_{k}\left(s_{k}\right) e^{-\frac{1}{2} \sum_{i, j=1}^{k}\left(\Sigma_{t}\right)_{i, j} s_{i} s_{j}} \\
& =\frac{1}{\sqrt{(2 \pi)^{k} \operatorname{det} \Sigma_{t}}} \int \mathrm{d} x_{1} \ldots \mathrm{d} x_{k} g_{1}\left(x_{1}\right) \ldots g_{k}\left(x_{k}\right) e^{-\frac{1}{2} \sum_{i, j=1}^{k}\left(\Sigma_{t}\right)_{i, j}^{-1} x_{i} x_{j}} .
\end{aligned}
$$

Summarizing the results from Lemmas 4-8, we finally obtain

$$
\begin{aligned}
& \mid \mathbb{E}_{\Psi_{N, t}}\left[g_{1}\left(\mathscr{O}_{1, N, t}\right) \ldots g_{k}\left(\mathscr{O}_{k, N, t}\right)\right] \\
& \quad-\frac{1}{\sqrt{(2 \pi)^{k} \operatorname{det} \Sigma_{t}}} \int \mathrm{d} x_{1} \ldots \mathrm{d} x_{k} g_{1}\left(x_{1}\right) \ldots g_{k}\left(x_{k}\right) e^{-\frac{1}{2} \sum_{i, j=1}^{k}\left(\Sigma_{t}\right)_{i, j}^{-1} x_{i} x_{j} \mid} \mid \\
& \leq C N^{-\gamma} \exp (C \exp (C|t|)) \\
& \quad \times \prod_{j=1}^{k} \int \mathrm{d} \tau\left|\widehat{g}_{j}(\tau)\right|\left(1+|\tau|^{2}\left\|O_{j}\right\|^{2}+N^{\gamma-1 / 2}|\tau|^{3}\left\|O_{j}\right\|^{3}+N^{\gamma-1}|\tau|^{4}\left\|O_{j}\right\|^{4}\right) .
\end{aligned}
$$

with $\gamma=\min \{\beta, 1-\beta\}$. This proves Theorem 1 . 
Acknowledgements Open access funding provided by Institute of Science and Technology (IST Austria). S.R. would like to thank Benjamin Schlein for many fruitful discussions.

\section{Compliance with ethical standards}

Conflict of interest On behalf of all authors, the corresponding author states that there is no conflict of interest.

Open Access This article is licensed under a Creative Commons Attribution 4.0 International License, which permits use, sharing, adaptation, distribution and reproduction in any medium or format, as long as you give appropriate credit to the original author(s) and the source, provide a link to the Creative Commons licence, and indicate if changes were made. The images or other third party material in this article are included in the article's Creative Commons licence, unless indicated otherwise in a credit line to the material. If material is not included in the article's Creative Commons licence and your intended use is not permitted by statutory regulation or exceeds the permitted use, you will need to obtain permission directly from the copyright holder. To view a copy of this licence, visit http://creativecommons.org/licenses/by/4.0/.

\section{References}

1. Adami, R., Golse, F., Teta, A.: Rigorous derivation of the cubic NLS in dimension one. J. Stat. Phys. 127(6), 1193-1220 (2007)

2. Ammari, Z., Falconi, M., Pawilowski, B.: On the rate of convergence for the mean field approximation of bosonic many-body quantum dynamics. Commun. Math. Sci. 14(5), 1417-1442 (2016)

3. Ammari, Z., Nier, F.: Mean field limit for bosons and propagation of Wigner measures. J. Math. Phys. 50(4), 042107 (2009). 16

4. Anapolitanos, I., Hott, M.: A simple proof of convergence to the Hartree dynamics in Sobolev trace norms. J. Math. Phys. 57(12), 122108 (2016). 8

5. Bardos, C., Golse, F., Mauser, N.J.: Weak coupling limit of the $N$-particle Schrödinger equation. Methods Appl. Anal. 7(2), 275-293 (2000)

6. Ben Arous, G., Kirkpatrick, K., Schlein, B.: A central limit theorem in many-body quantum dynamics, Commun. Math. Phys. 321(2), 371-417 (2013)

7. Benedikter, N., de Oliveira, G., Schlein, B.: Quantitative derivation of the Gross-Pitaevskii equation. Commun. Pure Appl. Math. 68(8), 1399-1482 (2015)

8. Benedikter, N., Porta, M., Schlein, B.: Effective evolution equations from quantum dynamics, Springer Briefs in Mathematical Physics, Springer (2016)

9. Boccato, C., Brennecke, C., Cenatiempo, S., Schlein, B.: The excitation spectrum of bose gases interacting through singular potentials. arXiv:1704.04819 (2017)

10. Boccato, C., Cenatiempo, S., Schlein, B.: Quantum many-body fluctuations around nonlinear Schrödinger dynamics. Ann. Henri Poincaré 18(1), 113-191 (2017)

11. Brennecke, C., Nam, P.T., Napiórkowski, M., Schlein, B.: Fluctuations of n-particle quantum dynamics around the nonlinear Schrödinger equation. Ann. Henri Poincaré (2018)

12. Brennecke, C., Schlein, B.: Gross-Pitaevskii dynamics for Bose-Einstein condensates. Anal. PDE 12(6), 1513-1596 (2019)

13. Buchholz, S., Saffirio, C., Schlein, B.: Multivariate central limit theorem in quantum dynamics. J. Stat. Phys. 154(1-2), 113-152 (2014)

14. Chen, L., Lee, J., Schlein, B.: Rate of convergence towards Hartree dynamics. J. Stat. Phys. 144(4), 872-903 (2011)

15. Chen, X., Holmer, J.: Correlation structures, many-body scattering processes, and the derivation of the Gross-Pitaevskii hierarchy. Int. Math. Res. Not. IMRN 10, 3051-3110 (2016)

16. Elgart, A., Schlein, B.: Mean field dynamics of boson stars. Commun. Pure Appl. Math. 60(4), 500-545 (2007)

17. Erdôs, L., Schlein, B., Yau, H.-T.: Derivation of the cubic non-linear Schrödinger equation from quantum dynamics of many-body systems. Invent. Math. 167(3), 515-614 (2007)

18. Erdős, L., Schlein, B., Yau, H.-T.: Rigorous derivation of the Gross-Pitaevskii equation with a large interaction potential. J. Am. Math. Soc. 22(4), 1099-1156 (2009) 
19. Erdős, L., Schlein, B., Yau, H.-T.: Derivation of the Gross-Pitaevskii equation for the dynamics of Bose-Einstein condensate. Ann. Math. 172(1), 291-370 (2010)

20. Erdôs, L., Yau, H.-T.: Derivation of the nonlinear Schrödinger equation from a many-body Coulomb system. Adv. Theor. Math. Phys. 5(6), 1169-1205 (2001)

21. Fröhlich, J., Knowles, A., Pizzo, A.: Atomism and quantization. J. Phys. A 40(12), 3033-3045 (2007)

22. Fröhlich, J., Knowles, A., Schwarz, S.: On the mean-field limit of bosons with Coulomb two-body interaction. Commun. Math. Phys. 288(3), 1023-1059 (2009)

23. Golse, F., On the dynamics of large particle systems in the mean field limit. Lecture notes for a course at the NDNS+Applied Dynamical systems summer school "Marcroscopic and large scale phenomena". Universiteit Twente, Enschede, The Netherlands, arXiv: 1301.5494 (2013)

24. Grillakis, M., Machedon, M.: Pair excitations and the mean field approximation of interacting bosons, II. Commun. Partial Differ. Equ. 42(1), 24-67 (2017)

25. Kirkpatrick, K., Schlein, B., Staffilani, G.: Derivation of the two-dimensional nonlinear Schrödinger equation from many body quantum dynamics. Am. J. Math. 133(1), 91-130 (2011)

26. Knwoles, A., Pickl, P.: Mean-field dynamics: singular potentials and rate of convergence. Commun. Math. Phys. 298(1), 101-138 (2010)

27. Lewin, M., Nam, P.T., Serfaty, S., Solovej, J.P.: Bogoliubov spectrum of interacting Bose gases. Commun. Pure Appl. Math. 68(3), 413-471 (2015)

28. Nam, P.T., Napiórkowski, M.: Bogoliubov correction to the mean-field dynamics of interacting bosons. Adv. Theor. Math. Phys. 21(3), 683-738 (2017)

29. Nam, P.T., Napiórkowski, M.: A note on the validity of Bogoliubov correction to mean-field dynamics. J. Math. Pures Appl. 108(5), 662-688 (2017)

30. Pickl, P.: Derivation of the time dependent Gross-Pitaevskii equation without positivity condition on the interaction. J. Stat. Phys. 140(1), 76-89 (2010)

31. Pickl, P.: Derivation of the time dependent Gross-Pitaevskii equation with external fields. Rev. Math. Phys. 27(1), 1550003 (2015). 45

32. Rademacher, S., Schlein, B.: Central limit theorem for Bose-Einstein condensates. J. Math. Phys. 60(7), 071902 (2019). 18

33. Schlein, B.: Derivation of effective equations from microscopic quantum dynamics. arXiv:0807.4307, Lecture notes for a course at ETH Zurich (2008)

34. Spohn, H.: Kinetic equations from Hamiltonian dynamics: Markovian limits. Rev. Modern Phys. 52(3), 569-615 (1980)

Publisher's Note Springer Nature remains neutral with regard to jurisdictional claims in published maps and institutional affiliations. 